\title{
The Max-Line-Formation Problem *
}

\author{
Jannik Castenow, Thorsten Götte, Till Knollmann, and Friedhelm Meyer auf der Heide \\ Heinz Nixdorf Institute and Department of Computer Science \\ Paderborn University, \{janniksu, thgoette, tillk, fmadh\}@mail.upb.de
}

\begin{abstract}
We consider $n$ robots with limited visibility: each robot can observe other robots only up to a constant distance denoted as the viewing range. The robots operate in discrete rounds that are either fully synchronous $\left(\mathcal{F}_{\text {SYNC }}\right)$ or semi-synchronized $\left(\mathcal{S}_{\text {SYNC }}\right)$. Most previously studied formation problems in this setting seek to bring the robots closer together (e.g., GATHERING or Chain-Formation). In this work, we introduce the MaX-Line-Formation problem, which has a contrary goal: to arrange the robots on a straight line of maximal length.

First, we prove that the problem is impossible to solve by robots with a constant sized circular viewing range. The impossibility holds under comparably strong assumptions: robots that agree on both axes of their local coordinate systems in $\mathcal{F}_{\text {SYNC. }}$ On the positive side, we show that the problem is solvable by robots with a constant square viewing range, i.e., the robots can observe other robots that lie within a constant-sized square centered at their position. In this case, the robots need to agree on only one axis of their local coordinate systems. We derive two algorithms: the first algorithm considers oblivious robots $(\mathcal{O B L O} \mathcal{L})$ and converges to the

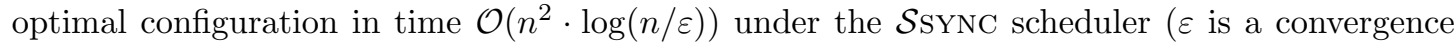
parameter). The other algorithm makes use of locally visible lights $(\mathcal{L U M \mathcal { I }})$. It is designed for the $\mathcal{F}_{\text {SYNC }}$ scheduler and can solve the problem exactly in optimal time $\Theta(n)$. We also argue how a combination of the two algorithms can solve the MAX-Line-Formation exactly in time $\mathcal{O}\left(n^{2}\right)$ under the $\mathcal{S}$ SYNC scheduler with the help of the $\mathcal{L U M \mathcal { I }}$ model.

Afterward, we show that both the algorithmic and the analysis techniques can also be applied to the Gathering and Chain-Formation problem: we introduce an algorithm with a reduced viewing range for GATHERING and give new and improved runtime bounds for the CHAIN-ForMATION problem.
\end{abstract}

\section{Introduction}

Robot formation tasks aim to arrange $n$ mobile robots in a specific formation. The robots are modeled as points in the Euclidean plane, and usually, the robot capabilities are very restricted. Robots are assumed to be externally identical (all robots have the same appearance), anonymous (no identifiers), autonomous (no central control) and homogeneous (all robots execute the same algorithm). Furthermore, the robots operate in discrete rounds denoted as LCM cycles. Each LCM cycle consists of three operations: Look, Compute and Move. During the Look operation, each robot takes a snapshot of its surroundings. Afterward, the robot computes a target point during Compute and finally moves there in the Move operation. With the additional assumptions that robots are silent (no communication) and oblivious (no memory of previous LCM cycles), this is

${ }^{*}$ This work was partially supported by the German Research Foundation (DFG) under the project number 453112019; ME 872/14-1. 
known as the $\mathcal{O B} \mathcal{L O} \mathcal{T}$ model [15]. The $\mathcal{L U M \mathcal { I }}$ model [10], on the contrary, does not demand the robots to be silent and oblivious. Instead, robots are equipped with a light that nearby robots (as well as the robot itself) can perceive. The light can have different colors, and thus, the robots obtain a constant-sized memory and can communicate state information to their neighbors. In addition to these core features, both models have a variety of freedom in some other assumptions; for instance, the LCM cycles might be fully synchronous $\left(\mathcal{F}_{\text {SYNC }}\right)$, semi-synchronous $(\mathcal{S}$ SYNC) or completely asynchronous ( $\mathcal{A S Y N C})$. All schedulers are assumed to be fair such that each robot can execute its LCM cycle infinitely often. Time is measured in epochs, i.e., the smallest number of rounds such that each robot has executed its LCM cycle at least once. In $\mathcal{F}$ SYNC an epoch is equal to one round.

Our focus lies on robots with limited visibility, i.e., each robot cannot perceive the entire swarm but only nearby robots. The terms connectivity range and viewing range are distinguished (see e.g., [5, 20]). Robots are connected to all robots up to a distance equal to their connectivity range and can see all robots within their viewing range (the viewing range is at least as large as the connectivity range). Initial configurations are connected w.r.t. the connectivity range and algorithms typically maintain this connectivity. The larger viewing range enhances the local information of the robots. Additionally, viewing and connectivity range can be circular or square. More precisely, a circular connectivity range of $c$ means that a robot is connected to all robots in the distance at most $c$ (all neighbors lie within the circle of radius $c$ around the robot). In contrast, the square connectivity range of $s c$ connects a robot $r$ to all other robots located within an axis aligned $2 s c \times 2 s c$-sized square centered at $r$. Similarly, circular and square viewing ranges are defined. In many applications, the connectivity and the viewing range are identical. The literature especially focusing on the runtime of formation algorithms often benefits from a viewing range that is larger than the connectivity range, see e.g., [1, 3, 4, 20].

Typical well-studied benchmark problems for robots with limited visibility are the GATHERING and the Chain-Formation problem. Gathering demands the robots to gather at a single, not predefined, position. Chain-Formation considers a chain of robots between two stationary outer robots: each inner robot has two identifiable neighbors (the neighborhoods are predefined and fixed). The goal is to arrange the robots on the line segment connecting the outer robots. Both Gathering and Chain-Formation can be characterized as contracting: the robots move closer together. Much less is known about formation tasks for robots with limited visibility that aim to achieve a contrary goal: to expand the robots' positions. One example is the UNIFORM-CIRCLEFORMATION problem in which $n$ robots are to move such that their positions form a regular polygon [11, 18. Another, very recent example and the main inspiration for this work is the MAX-CHAINFormation problem [5]. The MaX-Chain-Formation problem is a variant of the Chain-ForMATION problem. The difference is that MAX-CHAin-Formation gives the outer robots the ability to move. The new goal is to transform the chain of robots with connectivity and viewing range $c$ into a straight line of length $(n-1) \cdot c$.

In this work, we introduce the MAX-Line-Formation problem. The goal is similar to the MAX-Chain-Formation problem: to move the robots with connectivity range $c$ such that their positions form a straight line of length $(n-1) \cdot c$. The difference is that MAX-Line-Formation does not consider predefined chain neighborhoods. Instead, robots can observe the positions of all robots within their viewing range and do not have any fixed neighbors. We analyze under which robot capabilities the problem is solvable, derive algorithms, and analyze their runtime.

Related Work Due to space constraints, we focus on robots that operate in the LCM model and results about Gathering, Chain-Formation and MAX-Chain-Formation with a particular focus on research that considers a runtime analysis of the proposed algorithms. For a very recent 
and comprehensive overview of different robot formation algorithms, we refer the reader to [14. Oblivious and disoriented robots $(\mathcal{O B L O} \mathcal{T})$, can solve GATHERING in $\mathcal{O}\left(n^{2}\right)$ rounds $(\mathcal{F}$ SYNC) with the GTC algorithm. GTC moves robots in each round towards the center of the smallest enclosing circle of their neighborhood [2, 9]. GTC achieves the currently best-known runtime for disoriented and oblivious robots in the Euclidean plane. Faster algorithms for disoriented robots could so far only be designed under the $\mathcal{L U M I}$ model. There are two algorithms for robots located on a twodimensional grid [1, 7]. Another algorithm for robots in the Euclidean plane that are connected in a closed chain topology [4] exists. When assuming the $\mathcal{O B L O} \mathcal{T}$ model and one axis agreement, an asymptotically optimal algorithm with runtime $\mathcal{O}(\Delta)$ has been introduced in [20]. The algorithm assumes a square connectivity range of 1 and a circular viewing range of $\sqrt{10}$. Notably, the algorithm even works with the same runtime guarantees under the $\mathcal{A}$ SYNC scheduler.

Chain-Formation has been initially introduced in [12. The authors introduce the GTM algorithm that moves each robot to the midpoint between its neighbors. For the $\mathcal{F}$ SYNC scheduler, a runtime of $\mathcal{O}\left(n^{2} \cdot \log (n / \varepsilon)\right)$ rounds has been proven. Later on, an almost matching lower bound (for the algorithm) of $\Omega\left(n^{2} \cdot \log (1 / \varepsilon)\right)$ has been derived [16]. Algorithms with stronger assumptions, e.g., the $\mathcal{L U M \mathcal { I }}$ model, are able to achieve better runtimes [13, 17].

Very recently, the MaX-Chain-Formation problem has been introduced [5]. Started in onedimensional configurations, the MAX-GTM algorithm has a runtime of $\mathcal{O}\left(n^{2} \cdot \log (n / \varepsilon)\right)$ and $\Omega\left(n^{2}\right.$.

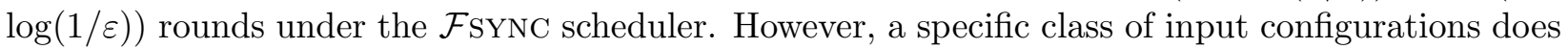
not converge to the optimal configuration. For two-dimensional configurations, only a convergence result is known. Additionally, for Gathering, Chain-Formation and Max-Chain-Formation, it is known that the problems can be solved optimally in a continuous time model [5, 8].

Our Contribution We introduce the Max-Line-Formation problem. The goal is to arrange $n$ robots with connectivity range $c$ on a straight line of length $(n-1) \cdot c$. We start with an impossibility result and prove that there are initial configurations for which the problem cannot be solved deterministically by robots with constant sized circular viewing and connectivity ranges. In addition, also no algorithm that converges to the optimal solution can exist for these configurations. The impossibility result even holds under strong assumptions: fully synchronized robots $\left(\mathcal{F}_{\mathrm{SYNC}}\right)$ that agree on both axes of their local coordinate systems. On the positive side, we show that the problem becomes solvable for robots with identical square connectivity and viewing ranges. While square connectivity and viewing ranges already have been proven to be useful to derive an efficient Gathering algorithm [20], the MAX-Line-Formation is the first known problem that can be solved under square viewing ranges but not under circular viewing ranges. Our algorithms require the robots to agree on only one axis of their local coordinate systems. We introduce two algorithms: The first algorithm considers the $\mathcal{O B L O} \mathcal{T}$ model and converges to the optimal solution in $\mathcal{O}\left(n^{2} \cdot \log (n / \varepsilon)\right)$ epochs under the $\mathcal{S}$ SYNC scheduler. The analysis idea is based on the sample variance of time inhomogeneous Markov chains (a concept similar to the mixing time of the time homogeneous case) inspired by [19]. Afterward, we show that enhancing the robots with the $\mathcal{L U M \mathcal { I }}$ model allows us to derive an improved algorithm, i.e., the algorithm solves the problem exactly while simultaneously improving the runtime. The algorithm considers the $\mathcal{F}_{\text {SYNC scheduler and solves the }}$ problem in $\Theta(n)$ epochs. The runtime is asymptotically optimal. Additionally, we argue that, with some additional synchronization, a combination of the two algorithms can solve the problem exactly with the help of lights in $\mathcal{O}\left(n^{2}\right)$ epochs under the $\mathcal{S}$ sYNC scheduler. Due to space constraints, only the high-level idea of the $\mathcal{S}_{\text {SYNC }}$ algorithm is contained in this version of the paper.

Our results compare to the MAX-GTM algorithm for MAX-CHAIN-Formation (which has the same goal but considers predefined and fixed chain neighborhoods) problem as follows: our runtime of the $\mathcal{O B} \mathcal{L O} \mathcal{T}$ algorithm holds under the $\mathcal{S}$ SYNC scheduler. For MAX-GTM, only runtimes in 
$\mathcal{F}_{\text {SYNC }}$ are known [5]. Additionally, our results about MAX-Line-Formation hold for every input configuration in which robots have distinct initial positions. For MAX-GTM, only a convergence result for a large class of configurations is known. Additionally, certain classes of configurations do not converge to the optimal configuration [5].

Moreover, we identify an interesting relation to Gathering and Chain-Formation. We first show that we can apply the main algorithmic idea of the $\Theta(n)$ algorithm to the GATHERING problem. More precisely, we derive an algorithm for the $\mathcal{O B L O} \mathcal{T}$ model that solves Gathering of $n$ robots that agree on one axis of their local coordinate systems in $\Theta(\Delta)$ epochs under the $\mathcal{F}$ SYNC scheduler, where $\Delta$ denotes the maximum distance of two robots in the initial configuration. 11 The algorithm uses a square viewing and connectivity range of 1 . Up to now, the best-known algorithm achieving the same runtime uses a square connectivity range of 1 and a circular viewing range of $\sqrt{10}[20]$. Thus, our algorithm closes the gap between viewing and connectivity range. Furthermore, we show how the analysis technique of the first algorithm (based on time inhomogeneous Markov chains) can also be applied for the Chain-Formation problem. In this context, disoriented robots (no agreement on the local coordinate systems) that are connected in a chain topology are assumed as well as a circular connectivity range and viewing range of 1 . We prove that the GTM algorithm [6, 13], in which each robot moves to the midpoint between its two direct neighbors in every round, converges to the optimal configuration in $\mathcal{O}\left(n^{2} \cdot \log (n / \varepsilon)\right)$ epochs assuming the $\mathcal{S}$ SYNC scheduler. For one-dimensional configurations (all robots are initially collinear) this is a significant improvement over the so far best known runtime bound of $\mathcal{O}\left(n^{5} \cdot \log (n / \varepsilon)\right)$ epochs for this algorithm [6]. For two-dimensional configurations, our result is the first runtime bound for the CHain-Formation problem derived for the $\mathcal{S}$ SYNC scheduler.

\section{Model \& Notation}

Time Model Robots operate in discrete LCM (Look, Compute, Move) cycles, denoted as rounds. Each robot takes a snapshot of its neighborhood during Look, computes a target point in Compute, and moves to this point in Move. We assume a rigid movement, robots always reach their target points during Move. The timing of the executions of the LCM cycles is either fully synchronous $(\mathcal{F}$ SYNC) or semi-synchronous $(\mathcal{S}$ SYNC), i.e., the cycles are synchronous, but only a subset of all robots participates. The executions are always fair: All robots execute their cycles infinitely often. Time is measured in epochs, i.e., the smallest number of rounds until each robot processes one complete LCM cycle. We assume that the execution starts in round $t_{0}$ and denote the first round of the $k$-th epoch by $t_{e_{k}}$. Thus, $t_{e_{1}}=t_{0}$.

Robot Model We consider $n$ robots $r_{1}, \ldots, r_{n}$ positioned in $\mathbb{R}^{2}$. Initially, the robots are located at pairwise distinct locations 2 . We assume a square connectivity and viewing range of 1 , i.e., two robots $r_{i}$ and $r_{j}$ are neighbors if $r_{j}$ is located inside of the $2 \times 2$-sized square centered at $r_{i}$ and vice versa. Note that 1 is only chosen for simplicity; it can be replaced by any constant $c$. The neighborhood of a robot $r_{i}$ (the set of all visible robots) in round $t$ is denoted by $N_{i}(t)$. The square connectivity graph in which two robots share an edge if they are neighbors is initially connected. Robots are assumed to be transparent and thus do not block the views between other robots. Moreover, the robots agree on one axis of their local coordinate systems. W.l.o.g. we assume that the robots agree on the $x$-axis. Thus, the robots have a common understanding of left and right, while up and down can be inverted. However, the robots agree on unit distance and can

\footnotetext{
${ }^{1} \Omega(\Delta)$ is a trivial lower bound since at least one of the robots forming the diameter $\Delta$ must cover a distance of at least $\frac{\Delta}{2}$ to obtain Gathering. Since the robots have limited visibility, this requires $\Omega(\Delta)$ rounds.

${ }^{2}$ Otherwise, the problem is deterministically unsolvable since multiplicities cannot be resolved if the robots are activated simultaneously.
} 
measure distances precisely. When considering the $\mathcal{O B} \mathcal{L O} \mathcal{T}$ model, the robots are also silent and oblivious.

For one algorithm, we consider the $\mathcal{L U M \mathcal { I }}$ model. Each robot is equipped with a constant number of lights $\ell_{1}, \ldots, \ell_{k}$ with color sets $C_{1}, \ldots, C_{k}$ and at every point in time, each light can have a single color out of its color set. ${ }^{3}$ Robots can perceive the lights of their neighbors during Look and can manipulate their light during Compute. Hence, if a robot $r_{i}$ decides to change its light color in round $t$, its neighbors can see this earliest in round $t+1$.

Notation The position of a robot $r_{j}$ in round $t$ is denoted by $p_{j}(t)=\left(x_{j}(t), y_{j}(t)\right)$ in a global coordinate system and by $p_{j}^{i}(t)=\left(x_{j}^{i}(t), y_{j}^{i}(t)\right)$ in the local coordinate system of $r_{i}$. Each robot lies in the center of its local coordinate system and thus $p_{i}^{i}(t)=(0,0)$. For a robot $r_{i}, r_{\ell}^{i}(t)$ denotes the leftmost robot of its neighborhood in round $t$. The position of $r_{\ell}^{i}(t)$ in the local coordinate system of $r_{i}$ in round $t$ is denoted by $p_{\ell}^{i}(t)=\left(x_{\ell}^{i}(t), y_{\ell}^{i}(t)\right)$. In case there are multiple such robots, $r_{\ell}^{i}(t)$ represents an arbitrary robot of all leftmost robots. Similarly, $r_{r}^{i}(t)$ and $p_{r}^{i}(t)$ are defined for the rightmost neighbor. Additionally, define $r_{+}^{i}(t)$ and $p_{+}^{i}(t)$ to be the closest neighbor above of $r_{i}$ and its position. Analogously, $r_{-}^{i}(t)$ and $p_{-}^{i}(t)$ is defined as the closest neighbor below and its position. In case no such robot exists, $r_{+}^{i}(t)=r_{i}$ and $r_{-}^{i}(t)=r_{i}$. For a vector $v$, we denote by $\widehat{v}$ the normalized vector $\frac{1}{\|v\|} v$.

Problem Statement MaX-Line-Formation demands to move $n$ robots with connectivity range $c$ such that their positions form a straight line of length $(n-1) \cdot c$. We say that an $(1-\varepsilon)$ approximation of the optimal configuration is reached if the positions form a straight line of length at least $(1-\varepsilon) \cdot(n-1) \cdot c$. During the entire execution of an algorithm, the connectivity graph has to remain connected.

\section{$3 \quad$ Impossibility Result \& Intuition about Square Ranges}

This section proves that MAX-Line-Formation is unsolvable with constant-sized circular viewing and connectivity ranges. Afterward, we give an intuition on how square ranges circumvent the impossibility.

\subsection{Impossibility with Circular Ranges}

Theorem 1. In the $\mathcal{O B L O} \mathcal{T}$ model, for every constant sized circular connectivity and viewing range, there exists an initial configuration with robots located at distinct positions such that the MAX-Line-Formation problem is unsolvable. Furthermore, no convergence algorithm can exist for these configurations. This holds for robots that agree on both axes of their local coordinate systems and the $\mathcal{F}$ SYNC scheduler.

Proof. Initially, we assume identical viewing and connectivity ranges. The arguments for viewing ranges that are larger than the connectivity range are analogous and can be found in Appendix A. Thus, we assume a circular viewing and connectivity range of $c$. We prove the claim by contradiction. We assume that there is an algorithm $\mathcal{M}$ that is able to solve the MAX-LinE-Formation problem. Next, we derive a combination of 2 initial configurations $C_{1}$ and $C_{2}$ and prove that if $\mathcal{M}$ is able to solve the problem starting in $C_{1}$, it cannot solve it starting in $C_{2}$. The configuration $C_{1}$ consists of three robots $r_{1}, r_{2}$ and $r_{3}$ at arbitrary (connected) positions. Since $\mathcal{M}$ is able to solve the problem,

\footnotetext{
${ }^{3}$ In the classical $\mathcal{L U} \mathcal{M I}$ model [10] each robot is equipped with a single light and color set. Our assumption of multiple lights and color sets can be transferred to the classical setting by choosing a single light with a color set of size at most $2^{\sum_{i=1}^{k}\left|C_{i}\right|}$.
} 

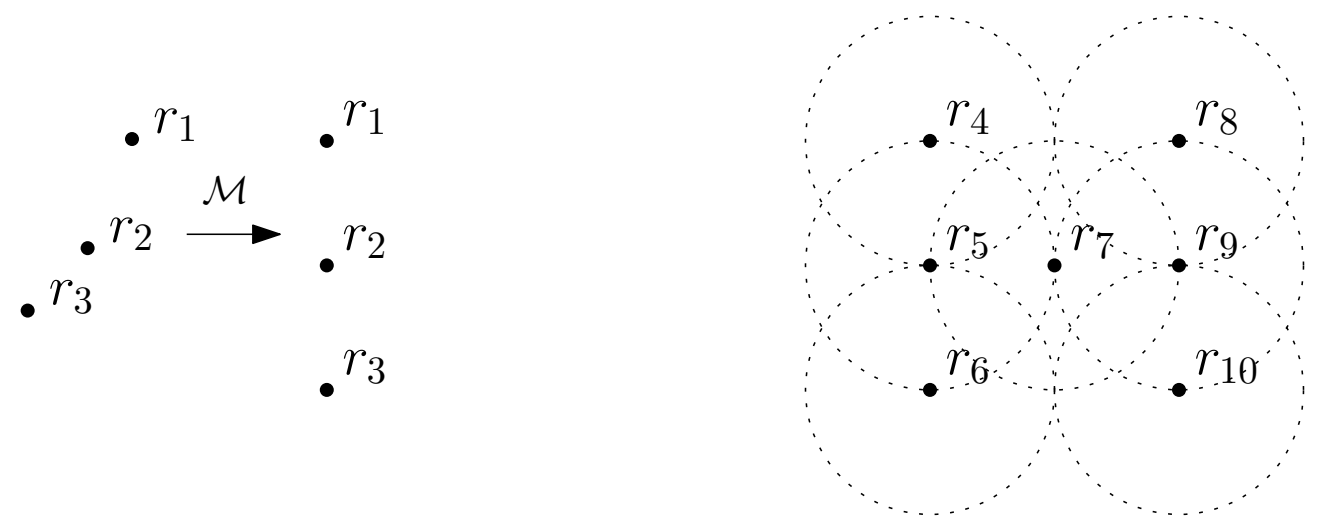

Figure 1: The config. $C_{1}$ transformed by $\mathcal{M}$.

Figure 2: The configuration $C_{2}$.

there is a time step $t_{f}$ such that the MAX-Line-Formation problem is solved. W.l.o.g. we assume that $r_{1}$ and $r_{3}$ are located at the end of the line and $p_{1}\left(t_{f}\right), p_{2}\left(t_{f}\right)$ and $p_{3}\left(t_{f}\right)$ form a line parallel to the $y$-axis (otherwise we could rename the robots and rotate the following configuration $C_{2}$ accordingly). More precisely, $p_{1}\left(t_{f}\right)-p_{2}\left(t_{f}\right)=p_{2}\left(t_{f}\right)-p_{3}\left(t_{f}\right)=(0, c)$. See Figure 1 for a depiction of the effects of $\mathcal{M}$ started in $C_{1}$.

The configuration $C_{2}$ consists of 7 robots, $r_{4}, \ldots, r_{10}$ located at the following positions in a global coordinate system (not known to the robots): $p_{4}(t)=(-c, c), p_{5}(t)=(-c, 0), p_{6}(t)=$ $(-c,-c), p_{7}(t)=(0,0), p_{8}(t)=(c, c), p_{9}(t)=(c, 0)$, and $p_{10}(t)=(c,-c)$. See Figure 2 for a visualization of the configuration. In $C_{2}, r_{4}$ can only see $r_{5}$ and is located in distance $c$ of $r_{5}$. Moreover, it holds $p_{4}(t)-p_{5}(t)=p_{1}\left(t_{f}\right)-p_{2}\left(t_{f}\right)$ and $\left\|p_{4}(t)-p_{5}(t)\right\|=c$. Thus, $\mathcal{M}$ is not allowed to move $r_{4}$ since $\mathcal{M}$ cannot distinguish $r_{1}$ in configuration $C_{1}$ after time $t_{f}$ and $r_{4}$ in configuration $C_{2}$. By similar arguments, $\mathcal{M}$ is also not allowed to move $r_{6}, r_{8}$ and $r_{10}$. Hence, the only remaining robots that could be moved by $\mathcal{M}$ are $r_{5}, r_{7}$ and $r_{9}$. However, also these robots are not allowed to move. Consider the robot $r_{5}$ which is located in maximum distance to $r_{4}, r_{6}$ and $r_{7}$. No matter where $r_{5}$ moves, it loses the connectivity to either $r_{4}$ or $r_{6}$ as these robots remain at their position. The same arguments hold for $r_{7}$ and $r_{9}$. It follows that $\mathcal{M}$ cannot solve the problem $C_{2}$, which contradicts the assumption.

\subsection{Intuition about Square Ranges}

Next, we argue why the proof of Theorem 1 does not hold when considering square viewing and connectivity ranges. Assume that the algorithm $\mathcal{M}$ transforms the configuration $C_{1}$ into a line that is parallel to the $y$-axis. Then, also the configuration $C_{2}$ is aligned with the $y$-axis. Still, the robots $r_{4}, r_{6}, r_{8}$ and $r_{10}$ are not allowed to move. The robots $r_{5}$ and $r_{9}$, however, gain the possibility to move horizontally. More precisely, $r_{5}$ is allowed to move to the right (a distance of at most 1 ) without losing the connectivity to $r_{4}$ and $r_{6}$ since the complete line segment connecting $r_{5}$ and $r_{7}$ is contained in the square viewing range of both $r_{4}$ and $r_{6}$. Similarly, $r_{9}$ can move to the left. See Figure 3 for a depiction of $C_{2}$ with square ranges instead of circular ones. Consequently, an algorithm solving the MAX-LinE-FORMATION with the help of square ranges should arrange the robots on a line parallel to the $y$-axis. The square ranges are only beneficial in case the local coordinate systems have the same orientation. In case the robots are disoriented, the same impossibility result of Section 3.1 also holds with square ranges. 


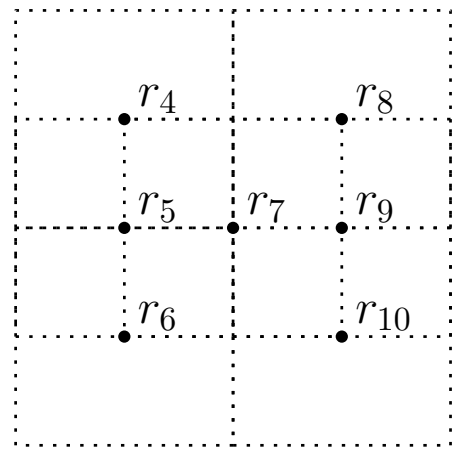

Figure 3: The configuration $C_{2}$ with square ranges instead of circular ones.

\section{$4 \quad \mathcal{O B} \mathcal{L O} \mathcal{T}$ Algorithm}

Based on the results of Section 3, MAX-LinE-FORMATION is unsolvable with circular viewing and connectivity ranges. In this section, we show that equipping the robots with square connectivity and viewing ranges allows us to design an algorithm that converges to the optimal solution. More precisely, we give an algorithm that converges to the optimal configuration assuming the $\mathcal{O B L O} \mathcal{T}$ model and a square viewing and connectivity range of 1 .

\subsection{Intuition}

The algorithm works in two phases. In the first phase, the positions of all robots are arranged on a straight line parallel to the $y$-axis. Afterward, the line is stretched in the second phase. Since the robots are oblivious and have limited visibility, robots cannot distinguish the phases and act upon their local view. Nevertheless, we will show that there is a time $t^{\prime}$ such that all robots have joined the second phase and will remain there for the rest of the execution.

Phase 1: A robot $r_{i}$ whose neighborhood has not yet formed a line parallel to the $y$-axis moves only if its position is rightmost in its neighborhood. Then, $r_{i}$ moves horizontally to the $x$-coordinate of its leftmost neighbor. If another robot already occupies this position, $r_{i}$ executes a slight vertical movement into the positive (from its local view) $y$-direction to avoid a collision. Collisions have to be avoided as they cannot be resolved deterministically. More precisely, if the robot is located topmost in its neighborhood, it moves a constant distance upwards. If the robot is not topmost, it determines the value $y_{\text {min }}^{i}$, the $y$-coordinate of its closest neighbor to the top. Afterwards, it moves $\frac{1}{3} y_{\text {min }}^{i}$ upwards. The factor of $\frac{1}{3}$ is essential since the robot with $y$-coordinate $y_{\min }^{i}$ might do the same movement while having a different understanding of up and down. Hence, a collision of the two robots is avoided.

Phase 2: In the second phase, all robots are located on the same line parallel to the $y$-axis, which can be seen as a particular case of the MAX-ChAin-Formation problem. Thus, the robots execute the MAX-GTM algorithm designed for MAX-ChAIn-Formation [5]: each inner robot (robots that have neighbors in each direction) move to the midpoint between their closest northern and their closest southern neighbor. Outer robots (at the end of the line) have to stretch the line and move as far as possible away from their closest neighbor without losing connectivity. Concretely, outer robots move as follows. Let $r_{1}$ be an outer robot and $r_{2}$ its closest neighbor and $v(t)=p_{1}(t)-p_{2}(t)$. Then, $r_{1}$ imagines a virtual robot $r_{v}$ at the position $p_{v}(t)=p_{1}(t)+\widehat{v}(t)$ and moves to $\frac{1}{2} p_{v}(t)+\frac{1}{2} p_{2}(t)$.

\subsection{Algorithm}

We define the following set of possibly colliding robots. For a robot $r_{i}$, define $C_{i}(t)=\left\{r_{j} \in\right.$ $N_{i}(t) \mid x_{j}^{i}(t)=0$ or $\left.x_{j}^{i}(t)=x_{\ell}^{i}(t)\right\}$. Now, $r_{\text {min }}^{i} \in C_{i}(t)$ is the robot with minimal $y_{\text {min }}^{i}(t)$ among all 
robots with $y_{\text {min }}^{i}(t)>0$. Thus, $r_{\text {min }}^{i}$ represents the robot lying above of $r_{i}$ (from $r_{i}$ 's view) that has the smallest $y$-coordinate among all robots in $C_{i}(t)$. If no such robot exists, define $y_{\text {min }}^{i}=\frac{1}{10}$. Algorithm 1 describes the movement of a robot $r_{i}$.

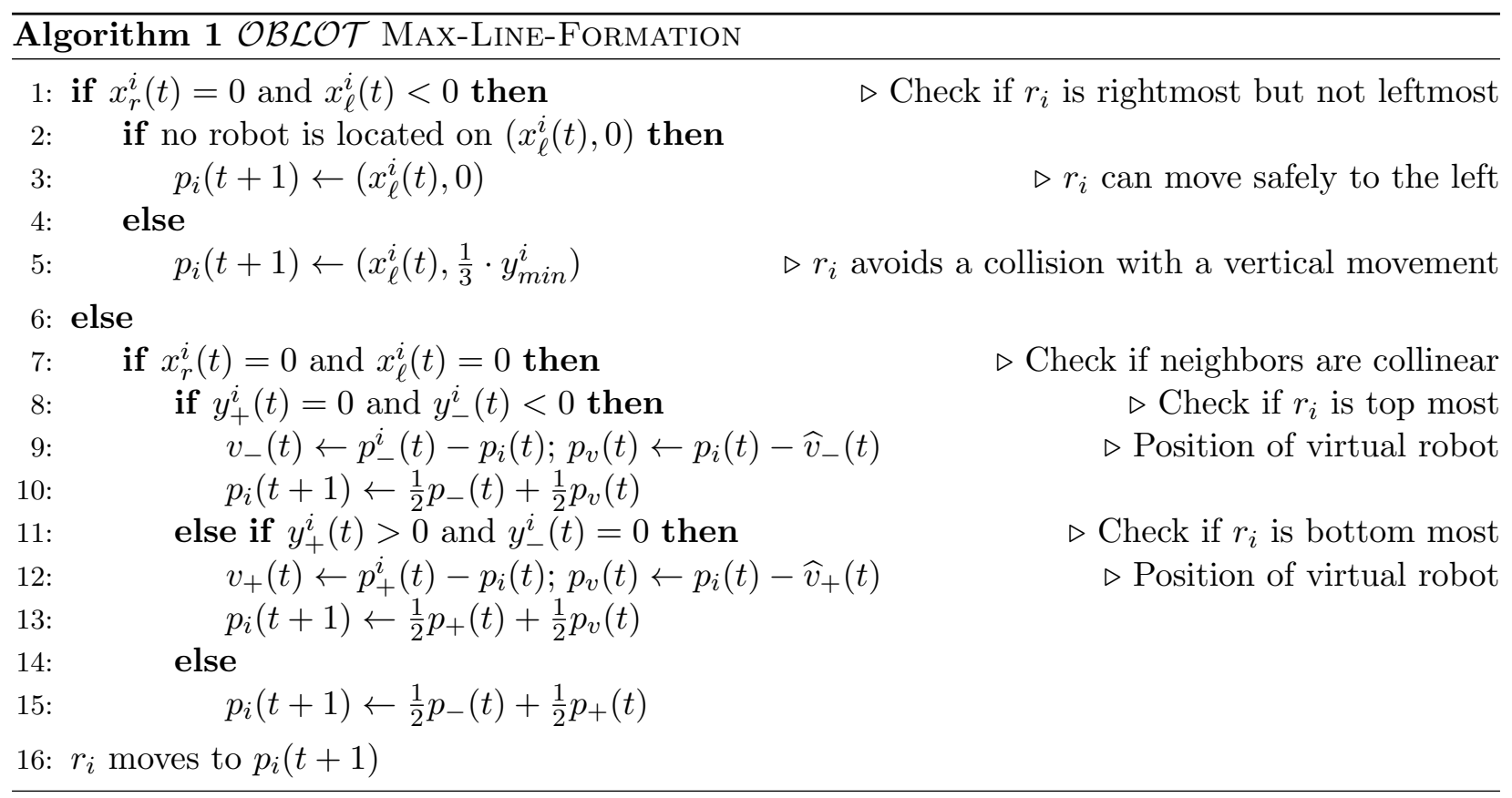

\subsection{Analysis}

Next, we introduce the analysis idea to prove the main theorem $($ Theorem 2 about the $\mathcal{O B L O} \mathcal{T}$ algorithm. Due to space constraints, all proofs are deferred to Appendix B.

Theorem 2. For every $0<\varepsilon<1$, after $\mathcal{O}\left(n^{2} \cdot \log (n / \varepsilon)\right)$ epochs, the robots have formed a line of length at least $(1-\varepsilon) \cdot(n-1)$.

First, we argue that the first phase of the algorithm ends after $\mathcal{O}\left(n^{2}\right)$ rounds.

Lemma 3. After $\mathcal{O}\left(n^{2}\right)$ epochs, all robots are located on distinct positions on the same vertical line parallel to the $y$-axis. Moreover, the configuration is connected.

Now, we can assume that the first phase is completed, and thus all robots are located on the same vertical line. W.l.o.g., we rename the robots such that $y_{1}(t) \leq y_{2}(t) \leq \ldots \leq y_{n}(t)$. Moreover, define $w_{1}(t)=1$ and $w_{i}(t)=y_{i}(t)-y_{i-1}(t)$ for $2 \leq i \leq n$. In addition, define $z_{i}(t)=\left(w_{i}(t)-w_{1}(t)\right)$. The algorithm is designed such that $\lim _{t \rightarrow \infty} w_{i}(t)=1$ for all $i$. To analyze this behavior, we consider the following function: $\Phi(t)=\sum_{i=2}^{n} z_{i}(t)^{2}$. The function $\Phi(t)$ is also known as the sample variance [19]. The name comes from a relation to time inhomogeneous Markov chains. Although the algorithm is deterministic, the behavior of the vectors $w_{i}(t)$ can be interpreted as a time inhomogeneous Markov Chain. The main course of our analysis is based on [19], where the authors analyzed a similar behavior in the context of the distributed averaging consensus problem. In this problem, there are $n$ agents, each having a numerical opinion. Every round, an agent gets to know some other opinions and updates its opinion to the average. Our application has one important difference: the values $w_{i}(t)$ do not average but converge to the fixed value $w_{1}(t)$. Hence, many parts of the proof in [19] have to be reworked and adapted to our application. First, we derive a bound on the change of $\Phi(t)$ 
between two epochs. Define $w_{\pi_{1}}\left(t_{e_{k}}\right), w_{\pi_{2}}\left(t_{e_{k}}\right), \ldots, w_{\pi_{n}}\left(t_{e_{k}}\right)$ to be the values $w_{i}\left(t_{e_{k}}\right)$ sorted from largest to smallest with ties broken arbitrarily.

Lemma 4. For any epoch $k, \Phi\left(t_{e_{k}}\right)-\Phi\left(t_{e_{k+1}}\right) \geq \frac{1}{4} \sum_{i=1}^{n-1}\left(w_{\pi_{i}}\left(t_{e_{k}}\right)-w_{\pi_{i+1}}\left(t_{e_{k}}\right)\right)^{2}$.

Based on Lemma 4, a lower bound on the relative change is derived.

Lemma 5. Suppose that $\Phi\left(t_{e_{k}}\right)>0$. Then, $\frac{\Phi\left(t_{e_{k}}\right)-\Phi\left(t_{e_{k+1}}\right)}{\Phi\left(t_{e_{k}}\right)} \geq \frac{1}{8 n^{2}}$.

A combination of Lemmas 4 and 5 yields the statement of Theorem 2.

\section{$5 \quad \mathcal{L U} \mathcal{M I}$ Algorithms}

In this section, we derive an algorithm that solves MAX-Line-Formation exactly with the help of the $\mathcal{L} \mathcal{M} \mathcal{I}$ model under the $\mathcal{F}_{\mathrm{SYNC}}$ scheduler (Section 5.1). The algorithm achieves an asymptotically optimal runtime of $\Theta(n)$ rounds. Additionally, in Section 5.2, we give an intuition about how a synchronization technique in combination with the $\mathcal{O B \mathcal { B O T }}$ (Section 4) and the $\mathcal{F}$ SYNC algorithm (Section 5.1) is able to solve MAX-Line-Formation exactly under the $\mathcal{S}$ SYNC scheduler in $\mathcal{O}\left(n^{2}\right)$ epochs.

\subsection{Fast Algorithm for the $\mathcal{F}$ sync scheduler}

The algorithm Algorithm 2 also works in two phases: In the first phase, all robots are arranged on a straight line parallel to the $y$-axis, and in the second phase, the line is stretched until it has maximal length. Compared to the $\mathcal{O B L O T}$ algorithm (Section 4), the algorithm uses different core ideas in both phases. In the first phase, all robots (instead of only the rightmost ones of their neighborhood) move to the left without losing connectivity - this is necessary to achieve a linear speedup of the first phase. The second phase makes use of lights to implement a sequential movement denoted as a run inspired by [1, 4, 7, 17]. For the sake of clarity and due to space constraints, we present a variant of the algorithm in which the robots still move to the left during the second phase. More precisely, after a linear number of rounds, the first phase ends, and the robots form a line parallel to the $y$-axis that continuously moves a distance of 1 to the left. Simultaneously, the robots stretch the line until it has maximal length. However, the line structure is always maintained such that MAX-Line-Formation is solved finally and remains solved (although the line keeps moving to the left). Moving continuously to the left can be removed from the algorithm with some additional effort; an intuition is given in Appendix C.

Phase 1: All robots move as far as possible to the left : each robot $r_{i}$ moves to the $x$-coordinate $x_{r}^{i}(t)-1$. Again, collision avoidance has to be ensured. While moving to $x_{r}^{i}(t)-1$, the robot $r_{i}$ could collide with every robot located on its local $x$-axis (since these robots potentially also want to move to the $x$-coordinate $\left.x_{r}^{i}(t)-1\right)$. The robot $r_{i}$ executes a vertical movement to avoid a collision. Based on the ordering of neighbors on the local $x$-axis, $r_{i}$ gets assigned a unique $y$-coordinate as follows: Define $Y_{i}(t)=\left\{r_{j} \in N_{i}(t) \mid y_{j}^{i}(t)=0\right\}$ and let $x_{\pi_{1}}(t), x_{\pi_{2}}(t), \ldots, x_{\pi_{\left|Y_{i}(t)\right|}}(t)$ be the $x$-coordinates of robots in $Y_{i}(t)$ in increasing order. Additionally, let $k_{i}(t) \in\left\{1, \ldots,\left|Y_{i}(t)\right|\right\}$ denote the position of $x_{i}(t)$ in the sorted sequence $x_{\pi_{1}}(t), x_{\pi_{2}}(t), \ldots, x_{\pi_{\left|Y_{i}(t)\right|}}(t)$. Furthermore, define $y_{\text {min }}^{i}(t)$ to be the minimal $y_{j}^{i}(t)$ of all $y_{j}^{i}(t)>0$ of robots $r_{j} \in N_{i}(t)$. If no such robot exists, define $y_{\min }^{i}(t)=\frac{1}{10}$ (any constant of size at most 1 works). Then, $r_{i}$ gets assigned the $y$-coordinate $\frac{k_{i}(t)-1}{\left|Y_{i}(t)\right|} \cdot \frac{1}{3} y_{\text {min }}^{i}(t)$. The factor $\frac{k_{i}(t)-1}{\left|Y_{i}(t)\right|}$ is unique for every robot on the local $x$-axis and the factor of $\frac{1}{3}$ is needed to prevent a collision with other robots that execute the same collision avoidance. 
Phase 2: For the second phase, lights are used. Assume w.l.o.g. that the robots are ordered along the $y$-axis, i.e., $y_{1}(t) \geq y_{2}(t) \geq \cdots \geq y_{n}(t)$. The core idea is a sequential movement started at $r_{1}$ and $r_{n}$ implemented with lights. Such a movement is called a run [1, 4, 17, 17]. Assume that a run starts in round $t$. Then, only $r_{1}$ and $r_{n}$ move. In round $t+1$, only $r_{2}$ and $r_{n-1}$ move and so on. A new run is started every three rounds.

Runs are realized with lights as follows. The first required light $\ell_{c}$ with color set $C_{c}=\{0,1,2\}$ is used as a round counter. Every round, all robots increment their light $\ell_{c}$. Whenever $\ell_{c}=2$ holds, both $r_{1}$ and $r_{n}$ activate a light $\ell_{m o v}$ with $C_{m o v}=\{0,1\}$ (the light is either active or inactive). An active light $\ell_{\text {mov }}$ enables the corresponding robot to move. Thus, in the next round, it holds $\ell_{c}=0$ and both $r_{1}$ and $r_{n}$ detect an active light $\ell_{\text {mov }}$. Both $r_{1}$ and $r_{n}$ now execute a movement (see below). Additionally, they deactivate the light $\ell_{\text {mov }}$ and activate a light $\ell_{\text {prev }}$ with color set $C_{\text {prev }}=\{0,1\}$ to remember the movement. Simultaneously, the robots $r_{2}$ and $r_{n-1}$ observe a neighbor on the $y$-axis with active light $\ell_{\text {mov }}\left(r_{1}\right.$ and $\left.r_{n}\right)$. Additionally, neither $r_{2}$ nor $r_{n-1}$ has activated $\ell_{\text {prev }}$. Hence, the robots activate $\ell_{\text {mov }}$ to continue the run. In the next round, $r_{1}$ and $r_{n}$ observe a neighbor with active light $\ell_{\text {mov }}$ but do not activate their own light $\ell_{\text {mov }}$ since $\ell_{\text {prev }}$ is active. Doing so ensures that the run keeps a fixed direction along the line.

Robots that have a run (the light $\ell_{m o v}$ is active) move as follows. In case $r_{1}$ has a run and not $r_{2}$ $(n>2), r_{1}$ moves in distance 1 vertically away from $r_{2}$. More formally, $p_{1}(t+1)=\left(x_{r}^{1}(t)-1,-\frac{y_{2}^{1}(t)}{\left|y_{2}^{1}(t)\right|}\right)$ (remember that in this variant the robots move also in phase 2 to the left). Similar, $r_{n}$ moves away from $r_{n-1}$ in distance 1 . In case a robot $r_{i}$ has a run that came from $r_{i-1}\left(r_{i-1}\right.$ has activated $\ell_{\text {prev }}$ and $r_{i}$ has activated $\left.\ell_{\text {mov }}\right)$ and $r_{i+1}$ does not have a run, $r_{i}$ moves in vertical distance 1 away from $r+1: p_{i}(t+1)=\left(x_{r}^{i}(t)-1,-\frac{y_{i+1}^{i}(t)}{\left|y_{i+1}^{i}(t)\right|}\right)$. Lastly, in case two neighboring robots have a run, for instance $r_{i}$ and $r_{i+1}$ have activated $\ell_{m o v}$ both move only a vertical distance of $\frac{1}{2}$ away from each other: $p_{i}(t+1)=\left(x_{r}^{i}(t)-1,-\frac{y_{i+1}^{i}(t)}{2\left|y_{i+1}^{i}(t)\right|}\right)$. The handling of the lights and the corresponding movement is depicted in Figure 4 .

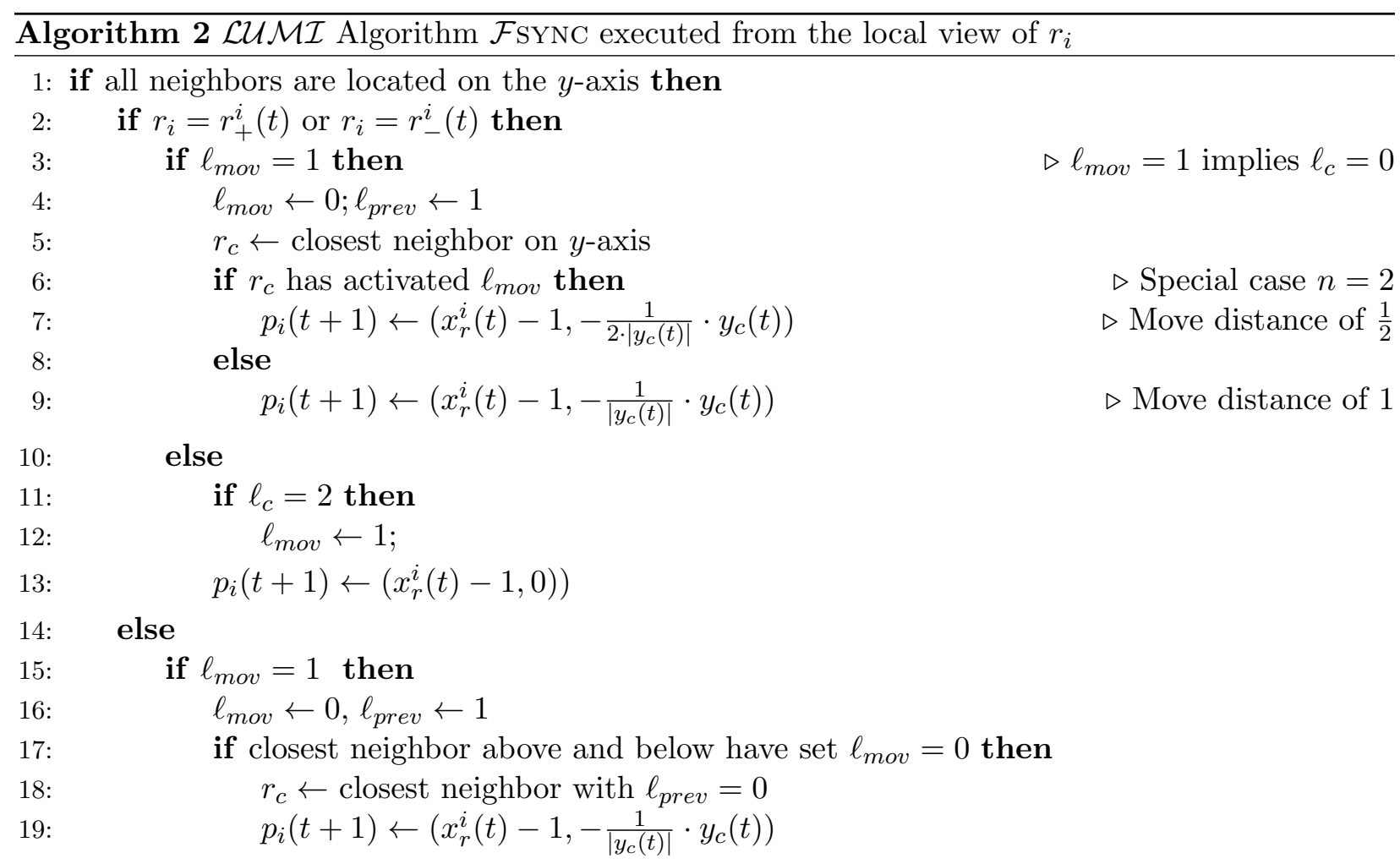




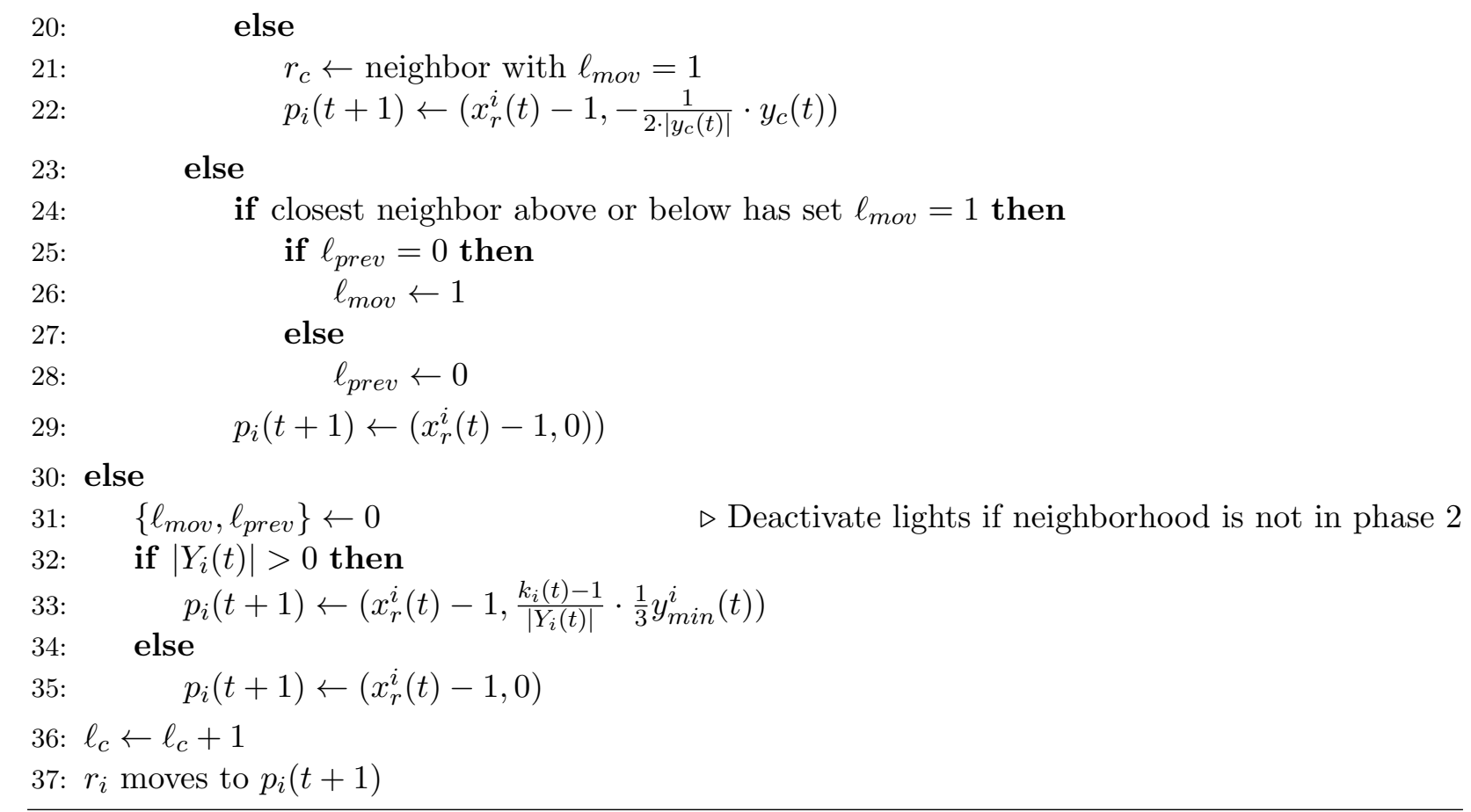

$\ell_{c}=2 \quad \ell_{c}=0 \quad \ell_{c}=1 \quad \ell_{c}=2 \quad \ell_{c}=0$

Figure 4: A square (cross) depicts a robot with active light $\ell_{\text {mov }}\left(\ell_{\text {prev }}\right)$. Time proceeds from left to right. In the first

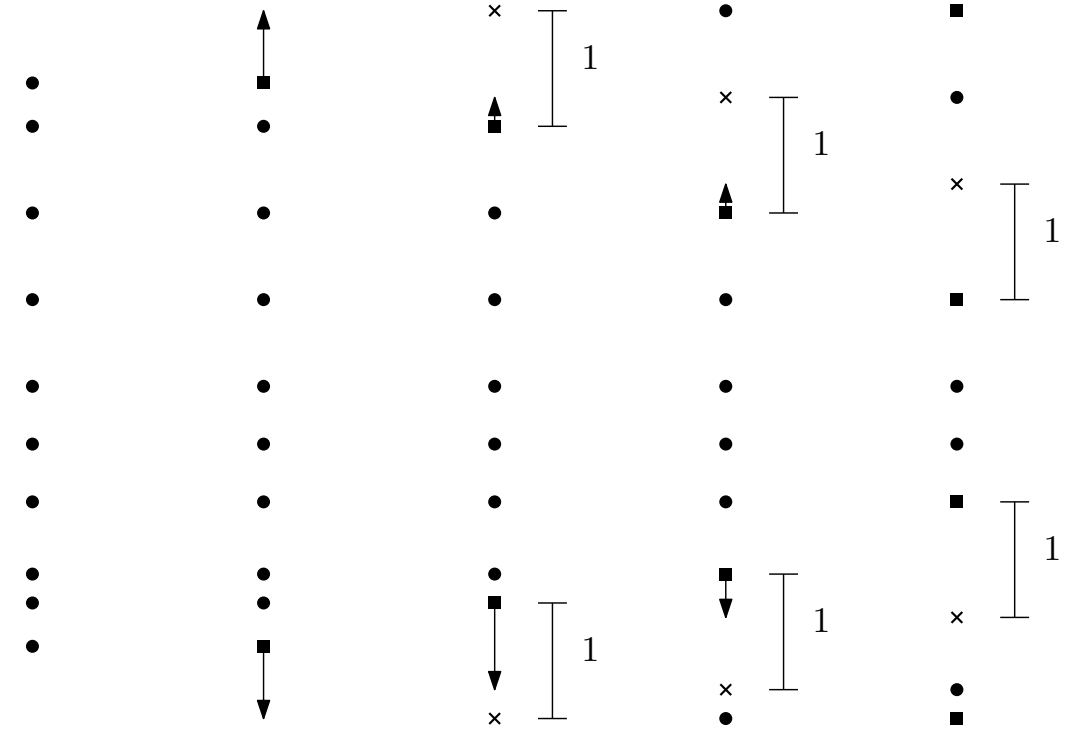
line it holds $\ell_{c}=2$ for all robots. In this round the top most and the bottom most robot activate $\ell_{\text {mov }}$. In the next round $\left(\ell_{c}=0\right)$, these two robots move in distance 1 of their neighbor (depicted by an arrow) and additionally deactivate $\ell_{m o v}$ while activating $\ell_{\text {prev }}$. Afterward $\left(\ell_{c}=1\right)$ the next two robots with active light $\ell_{\text {mov }}$ move in distance 1 of their next neighbor.

Analysis: In the analysis Appendix D, it is proven that after a linear number of rounds, the first phase ends (and thus, the robots have formed a line parallel to the $y$-axis). As a part of the proof, it is proven that no collisions occur, and the connectivity is always maintained. Moreover, it is proven that as soon as phase 2 is reached, the robots remain in phase 2 (following from the algorithm's description). Afterward, the runs of the second phase are analyzed. The first run ensures that after $\mathcal{O}(n)$ rounds, the robots $r_{\lfloor n / 2\rfloor}$ and $r_{\lfloor n / 2\rfloor+1}$ have a vertical distance of 1 . The second run ensures the same both for $r_{\lfloor n / 2\rfloor-1}$ and $r_{\lfloor n / 2\rfloor}$ as well as $r_{\lfloor n / 2\rfloor+1}$ and $r_{\lfloor n / 2\rfloor+2}$. Hence, after $\mathcal{O}(n)$ runs, the line reaches maximal length. Since each 3 rounds, a new run is started, and 
each run proceeds one robot per round, the linear runtime follows.

Lemma 6. After $\mathcal{O}(n)$ epochs, all robots are located on distinct positions on the same vertical line parallel to the $y$-axis. Moreover, the configuration is connected.

Theorem 7. After $\mathcal{O}(n)$ epochs, the robots have solved MAX-Line-Formation.

The algorithm can be implemented in the classical $\mathcal{L U M \mathcal { I }}$ model with a single light having 9 colors. Observe that no robot ever activates the lights $\ell_{\text {prev }}$ and $\ell_{\text {mov }}$ at the same time. Thus, for each robot, it always holds: either $\ell_{\text {prev }}, \ell_{\text {mov }}$ or none of both are activated. Additionally, each robot counts rounds with the light $\ell_{c}$ requiring 3 colors. Hence, the total number of required colors is 9: 3 colors of $\ell_{c}$, each combined with 3 possible cases for the lights $\ell_{\text {mov }}$ and $\ell_{\text {prev }}$.

\subsection{Ssync Scheduler}

The first phase of the $\mathcal{S}_{\mathrm{SYNC}}$ algorithm is identical to the first phase of the $\mathcal{O B} \mathcal{L O} \mathcal{T}$ algorithm (Section 4): Each robot that is rightmost in its neighborhood moves horizontally to the $x$-coordinate of its leftmost neighbor. In case this position is already occupied, a slight vertical movement is used to avoid collisions. The main idea of the second phase is the sequential movement (run) of Algorithm 2. Due to the $\mathcal{S}$ SYNC scheduler, an additional synchronization procedure needs to be added. In $\mathcal{F}$ SYNC, a robot with active light $\ell_{\text {mov }}$ can always be sure that the neighbors observe and adapt the light. Since only a subset of robots is active in every round in $\mathcal{S}$ sYNC, the light $\ell_{\text {mov }}$ might not be seen, and thus, the run stops. To overcome this, we add a synchronization done with the light $\ell_{c}$. In contrast to the $\mathcal{F}$ SYNC algorithm, the robots do not increment the light in every round they become active. Instead, each run gets associated with a color of the light $\ell_{c}$. More precisely, the main idea is as follows. Assume that the robots have already formed a line parallel to the $y$-axis. Moreover, we rename the robots such that $y_{1}(t) \leq y_{2}(t) \leq \cdots \leq y_{n}(t)$. Additionally, assume the configuration is well-initialized, i.e. all robots have set $\ell_{c}=0$. We describe the procedure from the view of $r_{1}$, it works analogously for $r_{n}$. We denote by $\ell_{i}\left(r_{j}\right)$ the color of $r_{j}$ 's light $\ell_{i}$ in round $t$ (the time parameter is omitted for readability). As soon as $r_{1}$ is activated, it observes $\ell_{c}\left(r_{2}\right)=\ell_{\text {prev }}\left(r_{2}\right)=\ell_{\text {mov }}\left(r_{2}\right)=0$. Then, $r_{1}$ activates $\ell_{\text {mov }}$. As soon as $r_{1}$ wakes up again, it executes its movement (it moves in distance 1 of $r_{2}$ ), deactivates $\ell_{\text {mov }}$, activates $\ell_{\text {prev }}$ and increments $\ell_{c}$ such that $\ell_{c}=1$. In the future, $r_{1}$ will only deactivate $\ell_{\text {prev }}$ in case it detects $\ell_{c}\left(r_{2}\right)=1$ (indicating that $r_{2}$ has taken over the run). Hence, as soon as $r_{2}$ is activated and detects $\ell_{c}\left(r_{1}\right)=\ell_{\text {prev }}\left(r_{1}\right)=1$ and $\ell_{c}\left(r_{3}\right)=\ell_{\text {prev }}\left(r_{3}\right)=\ell_{\text {mov }}\left(r_{3}\right)=0$, it will activate $\ell_{\text {mov }}$. Upon its next activation, $r_{2}$ executes its movement, deactivates $\ell_{\text {mov }}$. activates $\ell_{\text {prev }}$ and increments $\ell_{c}$. As soon as two neighboring robots have activated $\ell_{\text {mov }}$ both move in distance $\frac{1}{2}$ away from each other and stop the run (exactly as in Algorithm 2). This way, the runs proceed along the line. To conclude, a robot $r_{j}$ only takes over a run from its neighbor $r_{j-1}$ in case $\ell_{c}\left(r_{j-1}\right)=\ell_{c}\left(r_{j}\right)+1$. Additionally, $r_{j}$ will only deactivate $\ell_{\text {prev }}$ as soon as $\ell_{c}\left(r_{j-1}\right) \geq \ell\left(r_{j}\right)$ and $\ell_{c}\left(r_{j+1}\right)=\ell_{c}\left(r_{j}\right)$.

Note that it might happen due to the limited visibility that some runs already start while the first phase is not completed. Hence, at the beginning of phase 2, not all robots might be initialized with the same color of the light $\ell_{c}$. In case a robot detects such a violation (e.g., the next robot that should take over the light $\ell_{\text {mov }}$ has a larger value of $\ell_{c}$ ), the usual movement is not executed. Instead, simply the light $\ell_{c}$ is incremented. Hence, for each constant number of runs, the light of one more robot is well-initialized, and the algorithm adjusts the colors of the lights $\ell_{c}$ in a self-stabilizing manner. All in all, the first phase has a runtime of $\mathcal{O}\left(n^{2}\right)$ epochs (Lemma 3), the second phase is after $\mathcal{O}(n)$ epochs well-initialized (arguments above) and completed after additional $\mathcal{O}(n)$ epochs (Theorem 7). The runtime of $\mathcal{O}\left(n^{2}\right)$ epochs follows. 


\section{Relation to Gathering and Chain-Formation}

Finally, we show that we can also apply the main ideas of our algorithms for the MAX-LinEFormation problem in the context of Gathering and Chain-Formation.

\subsection{Gathering}

We consider robots in the $\mathcal{O B L O} \mathcal{T}$ model that agree on one axis of their local coordinate systems and operate under the $\mathcal{F}$ SYNC scheduler. Define $\Delta$ to be the maximal distance of two robots in the initial configuration in round $t_{0}$. Moreover, $\Delta_{x}$ denotes $\max _{i, j}\left|x_{i}\left(t_{0}\right)-x_{j}\left(t_{0}\right)\right|$ and analogously $\Delta_{y}$ denotes $\max _{i, j}\left|y_{i}\left(t_{0}\right)-y_{j}\left(t_{0}\right)\right|$. Observe that $\Delta_{x} \in \mathcal{O}(\Delta)$ and $\Delta_{y} \in \mathcal{O}(\Delta)$. The core idea of the GATHERING algorithm Algorithm 3 is as follows: to use the first phase of Algorithm 2 presented in Section 5.1 to arrange the robots on a vertical line fast. In this phase, every robot moves as far as possible to the left. While in Section 5.1, collisions have to be avoided, this is not necessary for GATHERING since collisions are desired to gather all robots on a single point. In Section 5.1 it has been proven that this phase requires $\mathcal{O}(n)$ epochs. We show with a slightly more elaborate argument that this phase requires only $\mathcal{O}(\Delta)$ epochs. The second phase squeezes the line to gather all robots and works as follows: robots at the end of the line move half the distance towards their farthest neighbor. All other robots move to the midpoint between their farthest neighbor above and their farthest neighbor below. The complete algorithm is contained in Algorithm 3. The following theorem states the $\mathcal{O}(\Delta)$ runtime, see Appendix E for a proof.

Theorem 8. GATHERING of $n$ robots agreeing on one axis of their local coordinate systems in the $\mathcal{O B} \mathcal{L O} \mathcal{T}$ model can be solved in $\mathcal{O}(\Delta)$ epochs under the $\mathcal{F}_{\text {SYNC }}$ scheduler.

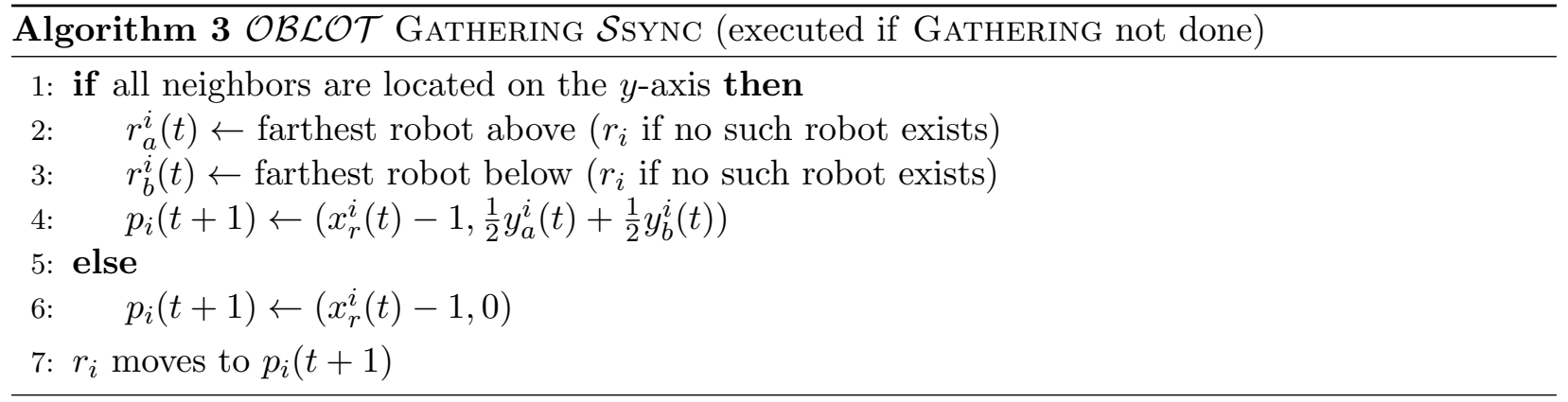

\subsection{Chain-Formation}

Lastly, we study the CHAin-Formation problem that considers disoriented robots. Additionally, the robots are connected in a chain topology: there are $n+2$ robots $r_{0}, r_{1}, \ldots, r_{n+1}$. The robots $r_{0}$ and $r_{n+1}$, denoted as outer robots, are stationary (they do not move). Every other robot $r_{i}$ has exactly two chain neighbors: $r_{i-1}$ and $r_{i+1}$ whose positions it can always observe. The robots have a circular connectivity and viewing range of 1 . Define by $w_{i}(t)=\left(w_{i}^{x}(t), w_{i}^{y}(t)\right)=p_{i}(t)-p_{i-1}(t)$ the vectors along the chain and $L(t)=\sum_{i=1}^{n+1}\left\|w_{i}(t)\right\|$. Additionally, $D=\left\|p_{0}(t)-p_{n+1}(t)\right\|$. The goal of the CHAin-Formation problem is to move the robots such that $L(t)=D$ and to distribute the robots uniformly along the line segment between $r_{0}$ and $r_{n+1}$. W.l.o.g,, assume that $r_{0}$ is positioned in the origin of a global coordinate system and $r_{n+1}$ on the positive $x$-axis in distance $D$ to $r_{0}$. Then, in the optimal configuration it holds $w_{i}(t)=w_{\infty}=\frac{D}{n+1}$ for $1 \leq i \leq n+1$. We say 
that an $\varepsilon$-approximation of the optimal configuration is reached in case $\left\|w_{i}(t)-w_{\infty}\right\| \leq \varepsilon$ for all $1 \leq i \leq n+1$.

For the problem, the GTM algorithm has been introduced [6, 13. The algorithm moves each robot in every round to the midpoint between its two direct neighbors. The GTM algorithm is very similar to the second phase of the $\mathcal{O B L O} \mathcal{T}$ algorithm Algorithm 1 presented in Section 4. Also, in Algorithm 1, robots that are not located at the end of the line move to the midpoint of their closest neighbors. In Algorithm 1, however, the robots at the of the line are moving to stretch the line. In contrast, the robots $r_{0}$ and $r_{n+1}$ of the Chain-Formation problem do not move. Nevertheless, we can apply a very similar analysis idea to the GTM algorithm: We prove convergence independently for $w_{i}^{x}(t)$ and $w_{i}^{y}(t)$. Since the arguments are identical, we concentrate on $w_{i}^{x}(t)$. Define $\bar{x}=\frac{1}{n+1} \cdot \sum_{i=1}^{n+1} w_{i}^{x}(t)$. Furthermore, define $z_{i}(t)=w_{i}^{x}(t)-\bar{x}$. The analysis is based on the following function: $\Phi_{2}(t)=\sum_{i=1}^{n+1} z_{i}(t)^{2}$ that can be analyzed in most parts analogously to $\Phi(t)$ in Section 4. See Appendix E.1 for a proof.

Theorem 9. For every $0<\varepsilon<1$, GTM reaches an $\varepsilon$-approximation of the optimal configuration in $\mathcal{O}\left(n^{2} \cdot \log (n / \varepsilon)\right)$ epochs under the $\mathcal{S}$ SYNC scheduler.

\section{Conclusion}

We have introduced the MAX-LinE-FORMATION problem and proven that the problem is impossible to solve with circular viewing and connectivity ranges. On the positive side, we have derived three algorithms for robots with square viewing and connectivity ranges. Several open questions remain: is it possible to solve the MAX-LINE-FORMATION exactly when considering oblivious robots $(\mathcal{O B L O} \mathcal{T})$ ? Is the derived runtime for the $\mathcal{O B L O} \mathcal{T}$ model tight or can there be a more efficient algorithm? The same question about lower bounds is also still open for the CHain-Formation and the Gathering problem. Can the problem be solved by disoriented robots (robots that do not agree on any axis)? For the last question, certainly square ranges do not help to solve the problem as the square ranges cannot be aligned according to a common axis.

\section{References}

[1] Abshoff, S., Cord-Landwehr, A., Fischer, M., Jung, D., Meyer auf der Heide, F.: Gathering a closed chain of robots on a grid. In: IPDPS. pp. 689-699. IEEE Computer Society (2016)

[2] Ando, H., Oasa, Y., Suzuki, I., Yamashita, M.: Distributed memoryless point convergence algorithm for mobile robots with limited visibility. IEEE Trans. Robotics Autom. 15(5), 818$828(1999)$

[3] Castenow, J., Fischer, M., Harbig, J., Jung, D., Meyer auf der Heide, F.: Gathering anonymous, oblivious robots on a grid. T. C. S. 815, 289-309 (2020)

[4] Castenow, J., Harbig, J., Jung, D., Knollmann, T., Meyer auf der Heide, F.: Brief announcement: Gathering in linear time: A closed chain of disoriented and luminous robots with limited visibility. In: SSS. LNCS, vol. 12514, pp. 60-64. Springer (2020)

[5] Castenow, J., Kling, P., Knollmann, T., Meyer auf der Heide, F.: A discrete and continuous study of the max-chain-formation problem - slow down to speed up. In: SSS. LNCS, vol. 12514, pp. 65-80. Springer (2020) 
[6] Cohen, R., Peleg, D.: Local spreading algorithms for autonomous robot systems. T. C. S. 399(1-2), 71-82 (2008)

[7] Cord-Landwehr, A., Fischer, M., Jung, D., Meyer auf der Heide, F.: Asymptotically optimal gathering on a grid. In: SPAA. pp. 301-312. ACM (2016)

[8] Degener, B., Kempkes, B., Kling, P., Meyer auf der Heide, F.: Linear and competitive strategies for continuous robot formation problems. ACM Trans. Parallel Comput. 2(1), 2:1-2:18 (2015)

[9] Degener, B., Kempkes, B., Langner, T., Meyer auf der Heide, F., Pietrzyk, P., Wattenhofer, R.: A tight runtime bound for synchronous gathering of autonomous robots with limited visibility. In: SPAA. pp. 139-148. ACM (2011)

[10] Di Luna, G.A., Viglietta, G.: Robots with lights. In: Distributed Computing by Mobile Entities, LNCS, vol. 11340, pp. 252-277. Springer (2019)

[11] Dutta, A., Chaudhuri, S.G., Datta, S., Mukhopadhyaya, K.: Circle formation by asynchronous fat robots with limited visibility. In: ICDCIT. LNCS, vol. 7154, pp. 83-93. Springer (2012)

[12] Dynia, M., Kutylowski, J., Lorek, P., Meyer auf der Heide, F.: Maintaining communication between an explorer and a base station. In: BICC. IFIP, vol. 216, pp. 137-146. Springer (2006)

[13] Dynia, M., Kutylowski, J., Meyer auf der Heide, F., Schrieb, J.: Local strategies for maintaining a chain of relay stations between an explorer and a base station. In: SPAA. pp. 260-269. ACM (2007)

[14] Flocchini, P., Prencipe, G., Santoro, N. (eds.): Distributed Computing by Mobile Entities, Current Research in Moving and Computing, LNCS, vol. 11340. Springer (2019)

[15] Flocchini, P., Prencipe, G., Santoro, N.: Moving and computing models: Robots. In: Distributed Computing by Mobile Entities, LNCS, vol. 11340, pp. 3-14. Springer (2019)

[16] Kling, P., Meyer auf der Heide, F.: Convergence of local communication chain strategies via linear transformations. In: SPAA. pp. 159-166. ACM (2011)

[17] Kutylowski, J., Meyer auf der Heide, F.: Optimal strategies for maintaining a chain of relays between an explorer and a base camp. T. C. S. 410(36), 3391-3405 (2009)

[18] Mondal, M., Chaudhuri, S.G.: Uniform circle formation by swarm robots under limited visibility. In: ICDCIT. LNCS, vol. 11969, pp. 420-428. Springer (2020)

[19] Nedic, A., Olshevsky, A., Ozdaglar, A.E., Tsitsiklis, J.N.: On distributed averaging algorithms and quantization effects. IEEE Trans. Autom. Control. 54(11), 2506-2517 (2009)

[20] Poudel, P., Sharma, G.: Universally optimal gathering under limited visibility. In: SSS. LNCS, vol. 10616, pp. 323-340. Springer (2017) 


\section{A Complete proof of Section 3}

Theorem 1. In the $\mathcal{O B L O} \mathcal{T}$ model, for every constant sized circular connectivity and viewing range, there exists an initial configuration with robots located at distinct positions such that the MAX-Line-Formation problem is unsolvable. Furthermore, no convergence algorithm can exist for these configurations. This holds for robots that agree on both axes of their local coordinate systems and the $\mathcal{F}_{\mathrm{SYNC}}$ scheduler.

Proof. Initially, we assume identical viewing and connectivity ranges. The arguments for viewing ranges that are larger than the connectivity range are analogous and can be found in Appendix A. Thus, we assume a circular viewing and connectivity range of $c$. We prove the claim by contradiction. We assume that there is an algorithm $\mathcal{M}$ that is able to solve the MAX-LinE-Formation problem. Next, we derive a combination of 2 initial configurations $C_{1}$ and $C_{2}$ and prove that if $\mathcal{M}$ is able to solve the problem starting in $C_{1}$, it cannot solve it starting in $C_{2}$. The configuration $C_{1}$ consists of three robots $r_{1}, r_{2}$ and $r_{3}$ at arbitrary (connected) positions. Since $\mathcal{M}$ is able to solve the problem, there is a time step $t_{f}$ such that the MAX-Line-Formation problem is solved. W.l.o.g. we assume that $r_{1}$ and $r_{3}$ are located at the end of the line and $p_{1}\left(t_{f}\right), p_{2}\left(t_{f}\right)$ and $p_{3}\left(t_{f}\right)$ form a line parallel to the $y$-axis (otherwise we could rename the robots and rotate the following configuration $C_{2}$ accordingly). More precisely, $p_{1}\left(t_{f}\right)-p_{2}\left(t_{f}\right)=p_{2}\left(t_{f}\right)-p_{3}\left(t_{f}\right)=(0, c)$. See Figure 1 for a depiction of the effects of $\mathcal{M}$ started in $C_{1}$.

The configuration $C_{2}$ consists of 7 robots, $r_{4}, \ldots, r_{10}$ located at the following positions in a global coordinate system (not known to the robots): $p_{4}(t)=(-c, c), p_{5}(t)=(-c, 0), p_{6}(t)=$ $(-c,-c), p_{7}(t)=(0,0), p_{8}(t)=(c, c), p_{9}(t)=(c, 0)$, and $p_{10}(t)=(c,-c)$. See Figure 2 for a visualization of the configuration. In $C_{2}, r_{4}$ can only see $r_{5}$ and is located in distance $c$ of $r_{5}$. Moreover, it holds $p_{4}(t)-p_{5}(t)=p_{1}\left(t_{f}\right)-p_{2}\left(t_{f}\right)$ and $\left\|p_{4}(t)-p_{5}(t)\right\|=c$. Thus, $\mathcal{M}$ is not allowed to move $r_{4}$ since $\mathcal{M}$ cannot distinguish $r_{1}$ in configuration $C_{1}$ after time $t_{f}$ and $r_{4}$ in configuration $C_{2}$. By similar arguments, $\mathcal{M}$ is also not allowed to move $r_{6}, r_{8}$ and $r_{10}$. Hence, the only remaining robots that could be moved by $\mathcal{M}$ are $r_{5}, r_{7}$ and $r_{9}$. However, also these robots are not allowed to move. Consider the robot $r_{5}$ which is located in maximum distance to $r_{4}, r_{6}$ and $r_{7}$. No matter where $r_{5}$ moves, it loses the connectivity to either $r_{4}$ or $r_{6}$ as these robots remain at their position. The same arguments hold for $r_{7}$ and $r_{9}$. It follows that $\mathcal{M}$ cannot solve the problem $C_{2}$, which contradicts the assumption.

Next, we consider a viewing range that is larger than the connectivity range but still a constant. W.l.o.g. we assume that there is a constant $\alpha>1$ such that the viewing range is of size $\alpha \cdot c$. The configuration is similar to before but the robots $r_{4}, r_{6}, r_{7}, r_{8}$ and $r_{10}$ are replaced by a line of $\lceil\alpha\rceil$ robots in maximum distance. More precisely, $r_{4}$ is replaced by $\lceil\alpha\rceil$ robots $r_{4,1}, r_{4,2}, \ldots, r_{4,\lceil\alpha\rceil}$ with $p_{4, j}(t)=(-\lceil\alpha\rceil \cdot c,(\lceil\alpha\rceil-j+1) \cdot c)$. Similarly, $r_{6}, r_{7}, r_{8}$ and $r_{10}$ are replaced. Hence, in total $\lceil\alpha\rceil \cdot 5+2$ robots are needed. See Figure 5 for a visualization. The configuration is designed such that $r_{4,1}, r_{6,2}, r_{81}$ and $r_{10,2}$ are not allowed to move since the configuration looks like the final configuration from their point of view. All other robots are not allowed to move since their movement would disconnect the connectivity graph. 


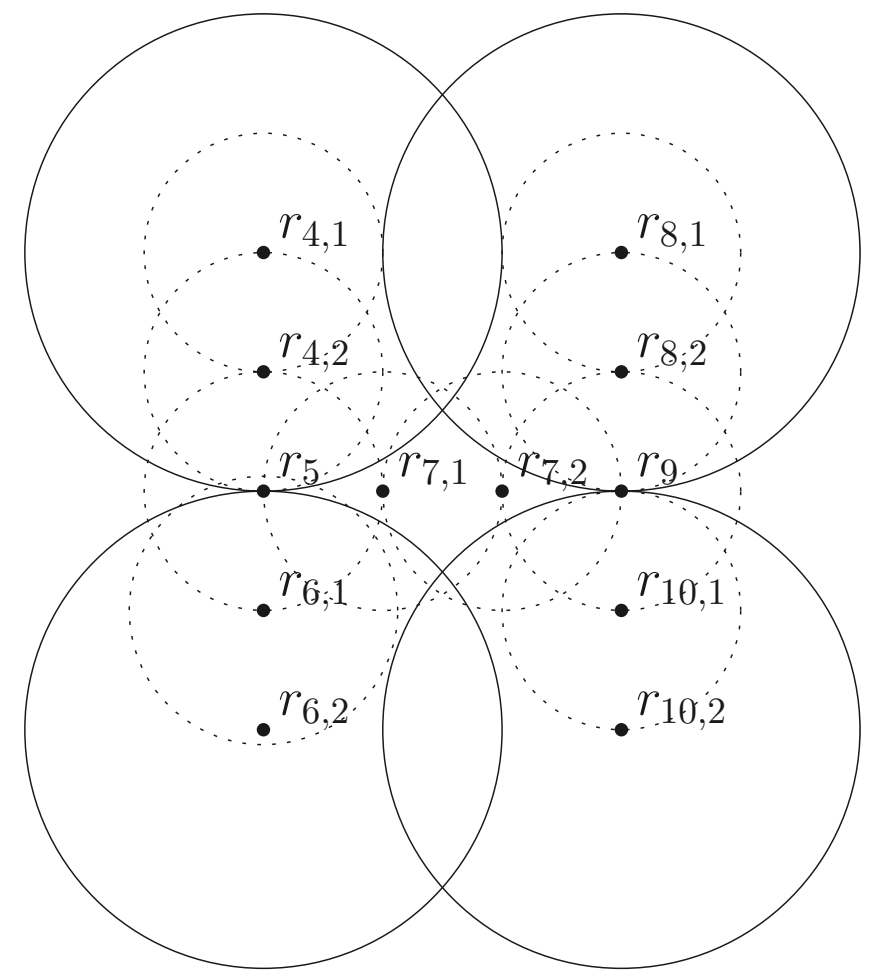

Figure 5: The configuration with $\alpha=2$ is depicted. The dotted circles represent the connectivity ranges of the robots. The solid circles depict the viewing ranges of selected robots (other viewing ranges are left out for the sake of clarity). 


\section{B Omitted Proofs of Section 4}

Lemma 3. After $\mathcal{O}\left(n^{2}\right)$ epochs, all robots are located on distinct positions on the same vertical line parallel to the $y$-axis. Moreover, the configuration is connected.

Proof. Initially, at most $n$ distinct $x$-coordinates that are occupied by robots exist. In every epoch, at least one robot that occupies the rightmost $x$-position moves to the left as the configuration is connected. This movement does not create any new $x$-position as the robot moves to the $x$ coordinate of its leftmost neighbor. Additionally, no robot moves to the right. Hence, after at most $n$ epochs, no robot occupies the rightmost $x$-coordinate anymore. Thus, after $\mathcal{O}\left(n^{2}\right)$ epochs, all robots are located on the same vertical line by applying the same argument inductively. The connectivity and non-existence of collisions follow from the algorithm's description.

We define $\tau_{i}(t)=1$ if and only if $r_{i}$ is active in round $t$. First of all, we derive formulas for the vectors $w_{i}(t+1)$. For each vector, we have to consider 4 cases: $\tau_{i}(t)=1$ and $\tau_{i-1}(t)=1$, $\tau_{i}(t)=1$ and $\tau_{i-1}(t)=0, \tau_{i}(t)=0$ and $\tau_{i-1}(t)=1$ and $\tau_{i}(t)=0$ and $\tau_{i-1}(t)=0$. Furthermore, $\mu_{i}^{-}(t)=\tau_{i-1}(t) \cdot\left(\tau_{i-1}(t)-\tau_{i}(t)\right)$ and $\mu_{i}^{+}(t)=\tau_{i}(t) \cdot\left(\tau_{i}(t)-\tau_{i-1}(t)\right)$. For the ease of notation, define $d_{i}^{-}(t)=\mu_{i}^{-}(t) \cdot\left(w_{i}(t)-w_{i-1}(t)\right)^{2}, d_{i}(t)=\tau_{i}(t) \cdot \tau_{i-1}(t) \cdot\left(w_{i-1}(t)-w_{i+1}(t)\right)^{2}$ and $d_{i}^{+}(t)=$ $\mu_{i}^{+}(t) \cdot\left(w_{i}(t)-w_{i+1}(t)\right)^{2}$. Observe that $d_{i}^{-}(t), d_{i}(t)$ and $d_{i}^{+}(t)$ are defined such that at most one of the three terms can be larger than 0 (the other ones are equal to 0 ). Lastly, define $w_{n+1}(t)=w_{1}(t)$.

Lemma 10. For any round $t$, it holds

$$
\Phi(t+1)=\Phi(t)-\frac{1}{4} \sum_{i=2}^{n} d_{i}^{-}(t)+d_{i}(t)+d_{i}^{+}(t) .
$$

Proof. Consider a vector $w_{i}(t)$ with $2<i<n$. Next, we calculate $w_{i}(t+1)$. There are 4 cases to consider: Case 1: $\tau_{i-1}(t)=1$ and $\tau_{i}(t)=1$, Case 2 and 3: $\tau_{i-1}(t)=1$ and $\tau_{i}(t)=0$ or vice versa and Case 4: $\tau_{i-1}(t)=0$ and $\tau_{i}(t)=0$. The following formulas can be easily verified:

1. Case 1: $w_{i}(t+1)=\frac{1}{2} w_{i-1}(t)+\frac{1}{2} w_{i+1}(t)$

2. Case 2: $w_{i}(t+1)=\frac{1}{2} w_{i-1}(t)+\frac{1}{2} w_{i}(t)$

3. Case 3: $w_{i}(t+1)=\frac{1}{2} w_{i}(t)+\frac{1}{2} w_{i+1}(t)$

4. Case 4: $w_{i}(t+1)=w_{i}(t)$

The formulas for the boundary vectors $w_{2}(t)$ and $w_{n}(t)$ are slightly different: Case 1: $\tau_{1}(t)=1$, $\tau_{2}(t)=1, \tau_{n-1}(t)=1, \tau_{n}(t)=1$, Case 2 and 3: $\tau_{1}(t)=1, \tau_{2}(t)=0, \tau_{n-1}(t)=0$ and $\tau_{n}(t)=1$ or vice versa and Case 4: $\tau_{1}(t)=0, \tau_{2}(t)=0, \tau_{n-1}(t)=0$ and $\tau_{n}(t)=0$.

1. Case 1: $w_{2}(t+1)=\frac{1}{2} w_{1}(t)+\frac{1}{2} w_{3}(t) ; w_{n}(t+1)=\frac{1}{2} w_{n-1}(t)+\frac{1}{2} w_{n+1}(t)$

2. Case 2: $w_{2}(t+1)=\frac{1}{2} w_{1}(t)+\frac{1}{2} w_{2}(t) ; w_{n}(t+1)=\frac{1}{2} w_{n}(t)+\frac{1}{2} w_{n+1}(t)$

3. Case 3: $w_{2}(t+1)=\frac{1}{2} w_{2}(t)+\frac{1}{2} w_{3}(t) ; w_{n}(t+1)=\frac{1}{2} w_{n-1}(t)+\frac{1}{2} w_{n}(t)$

4. Case 4: $w_{2}(t+1)=w_{2}(t) ; w_{n}(t+1)=w_{n}(t)$

Next, we derive a formula for $z_{i}(t+1)^{2}$ for $2<i<n$. Observe first $z_{i}(t)^{2}=\left(w_{i}(t)-1\right)^{2}=$ $w_{i}(t)^{2}-2 \cdot w_{i}(t)+1$. 
1. Case 1: $z_{i}(t+1)^{2}=\left(\frac{1}{2} w_{i-1}(t)+\frac{1}{2} w_{i+1}(t)-1\right)^{2}=\frac{1}{4} w_{i-1}(t)^{2}+\frac{1}{4} w_{i+1}(t)^{2}+\frac{w_{i-1}(t) \cdot w_{i+1}(t)}{2}-$ $w_{i-1}(t)-w_{i+1}(t)+1$

2. Case 2: $z_{i}(t+1)^{2}=\frac{1}{4} w_{i-1}(t)^{2}+\frac{1}{4} w_{i}(t)^{2}+\frac{w_{i-1}(t) \cdot w_{i}(t)}{2}-w_{i-1}(t)-w_{i}(t)+1$

3. Case 3: $z_{i}(t+1)^{2}=\frac{1}{4} w_{i}(t)^{2}+\frac{1}{4} w_{i+1}(t)^{2}+\frac{w_{i}(t) \cdot w_{i+1}(t)}{2}-w_{i}(t)-w_{i+1}(t)+1$

4. Case 4: $z_{i}(t+1)^{2}=z_{i}(t)^{2}$

Similar formulas can be derived for $z_{2}(t+1)$ and $z_{n}(t+1)$. Since at most one of the three terms $d_{i}^{-}(t), d_{i}^{+}(t)$ and $d_{i}(t)$ is positive, and each $w_{i}(t)$ occurs exactly twice in all $z_{i}(t+1)$ 's, the lemma follows.

Lemma 4. For any epoch $k, \Phi\left(t_{e_{k}}\right)-\Phi\left(t_{e_{k+1}}\right) \geq \frac{1}{4} \sum_{i=1}^{n-1}\left(w_{\pi_{i}}\left(t_{e_{k}}\right)-w_{\pi_{i+1}}\left(t_{e_{k}}\right)\right)^{2}$.

Proof. By Lemma 10, we obtain

$$
\Phi\left(t_{e_{k}}\right)-\Phi\left(t_{e_{k+1}}\right) \geq \frac{1}{4} \cdot \sum_{t=t_{e_{k}}}^{t_{e_{k+1}}} \sum_{i=2}^{n} d_{i}^{-}(t)+d_{i}(t)+d_{i}^{+}(t) .
$$

The first part of the proof deals with finding a lower bound for any $d_{i}^{-}(t)+d_{i}(t)+d_{i}^{+}(t)$ given that at least one of the terms is larger than 0 (at most one of the three terms is positive). The lower bound, however, depends on the sorted sequence $w_{\pi_{1}}(t), \ldots w_{\pi_{n}}(t)$. Since we lose much structure due to the sorting, some definitions are needed. Let $\pi$ be the function that maps the indices of $w_{1}\left(t_{e_{k}}\right), \ldots, w_{n}\left(t_{e_{k}}\right)$ into the sorted sequence $w_{\pi_{1}}\left(t_{e_{k}}\right), \ldots, w_{\pi_{n}}\left(t_{e_{k}}\right)$ and $\pi^{-1}$ its inverse. More precisely, for instance $\pi(i)=\pi_{f}$ if and only if $w_{i}\left(t_{e_{k}}\right)=w_{\pi_{f}}\left(t_{e_{k}}\right)$. Furthermore, define $\sigma_{m, i, j}(t)=1$ if and only if one of the following cases is fulfilled:

1. $d_{\pi_{m}}(t)>0$ and $\pi\left(\pi^{-1}\left(\pi_{m}\right)-1\right)=\pi_{i}$ and $\pi\left(\pi^{-1}\left(\pi_{m}\right)+1\right)=\pi_{j}$ or vice versa

2. $d_{\pi_{m}}^{-}(t)>0$ and $\pi\left(\pi^{-1}\left(\pi_{m}\right)-1\right)=\pi_{i}$ and $\pi\left(\pi^{-1}\left(\pi_{m}\right)\right)=\pi_{j}$ or vice versa

3. $d_{\pi_{m}}^{+}(t)>0$ and $\pi\left(\pi^{-1}\left(\pi_{m}\right)\right)=\pi_{i}$ and $\pi\left(\pi^{-1}\left(\pi_{m}\right)+1\right)=\pi_{j}$ or vice versa

Due to the sorting, we lose the nice property that only neighboring $w_{i}(t)$ 's are involved in $d_{\pi_{m}}(t)$, $d_{\pi_{m}}^{-}(t)$ and $d_{\pi_{m}}^{+}(t)$. For instance in case $d_{\pi_{m}}(t)>0$ we cannot conclude that $w_{\pi_{m}-1}(t)$ and $w_{\pi_{m}+1}(t)$ ar involved. Thus, intuitively, $\sigma_{m, i, j}(t)=1$ if and only if $d_{\pi_{m}}(t), d_{\pi_{m}}^{-}(t)$ or a $d_{\pi_{m}}^{+}(t)$ is larger than 0 and both $w_{\pi_{i}}(t)$ and $w_{\pi_{j}}(t)$ are involved.

Next, define $t_{\ell}(1 \leq \ell \leq n)$ to be the first round larger than or equal to $t_{e_{k}}$ such that there exists three indices $\pi_{i}, \pi_{j}$ and $\pi_{m}\left(\pi_{i} \neq \pi_{j}\right.$ but $\pi_{i}=\pi_{m}$ or $\pi_{j}=\pi_{m}$ might hold $)$ with $\pi_{i} \leq \pi_{\ell}<\pi_{j}$ (or vice versa) and $\sigma_{m, i, j}(t)=1$. In other words, $t_{\ell}$ denotes the first round in which the values $w_{\pi_{1}}\left(t_{e_{k}}\right), \ldots, w_{\pi_{\ell}}\left(t_{e_{k}}\right)$ and $w_{\pi_{\ell+1}}\left(t_{e_{k}}\right), \ldots, w_{\pi_{n}}\left(t_{e_{k}}\right)$ influence each other. By influencing each other, we mean that $w_{\pi_{m}}(t+1)=\frac{1}{2} w_{\pi_{i}}(t)+\frac{1}{2} w_{\pi_{j}}(t)$, since either $d_{\pi_{m}}(t)>0, d_{\pi_{m}}^{-}(t)>0$ or $d_{\pi_{m}}^{+}(t)>04^{4}$

For all $t \in\left\{t_{e_{k}}, \ldots, t_{e_{k+1}}\right\}$ let $L(t)=\left\{\ell \mid t_{\ell}=t\right\}$, i.e. $L(t)$ represents all indices $\ell$ at time $t$ such that the two sets $\left\{w_{\pi_{1}}\left(t_{e_{k}}\right), \ldots w_{\pi_{\ell}}\left(t_{e_{k}}\right)\right\}$ and $\left\{w_{\pi_{\ell+1}}\left(t_{e_{k}}\right), \ldots, w_{\pi_{n}}\left(t_{e_{k}}\right)\right\}$ influence each other for the first time.

\footnotetext{
${ }^{4}$ In the context of averaging consensus each index $1, \ldots, n$ corresponds a node in the graph. Thus, the index $\ell$ can be interpreted as a cut in the graph and the time $t_{\ell}$ as the first time with a communication across the cut represented by $\ell$.
} 
Now, we define all pairs of indices $\pi_{i}, \pi_{j}$ at time $t$ such that there exists an $\pi_{\ell}$ with $\pi_{i} \leq \pi_{\ell}<\pi_{j}$ and $\sigma_{\ell, i, j}(t)=1: C_{\ell}(t)=\left\{\left\{\pi_{i}, \pi_{j}\right\} \mid \pi_{i} \leq \pi_{\ell}<\pi_{j}\right.$ and $\left.\sigma_{\ell, i, j}(t)=1\right\}$. Lastly, define for fixed $i, j$ and $t: F_{i j}(t)=\left\{\ell \in L(t) \mid\left\{\pi_{i}, \pi_{j}\right\} \in C_{\ell}(t)\right\}$.

Fix some $\pi_{i}$ and $\pi_{j}$ with $\pi_{i}<\pi_{j}$ and a round $t$ such that $\left|F_{i j}(t)\right|>0$. Let $F_{i j}(t)=\left\{\ell_{1}, \ldots, \ell_{k}\right\}$ sorted in increasing order. Since $\ell_{1} \in L(t)$, it holds by definition that there exists no round $t^{\prime} \in\left[t_{e_{k}}, \ldots, t\right]$ and an index $\pi_{m}$ with $\pi_{i} \leq \pi_{m}<\pi_{j}$ such $\sigma_{m, i, j}\left(t^{\prime}\right)=1$. It follows $w_{\pi_{i}}(t) \geq w_{\pi_{\ell_{1}}}\left(t_{e_{k}}\right)$ (since $w_{\pi_{i}}(t)$ was so far only influenced by elements of the set $w_{\pi_{1}}\left(t_{e_{k}}\right), \ldots, w_{\pi_{\ell}}\left(t_{e_{k}}\right)$ which are all larger or equal to $w_{\pi_{\ell}}\left(t_{e_{k}}\right)$ ). Similarly, one can argue $w_{\pi_{j}}(t) \leq w_{\pi_{\ell_{k}+1}}\left(t_{e_{k}}\right)$. Hence, we can conclude

$$
w_{\pi_{i}}(t)-w_{\pi_{j}}(t) \geq w_{\pi_{\ell_{1}}}\left(t_{e_{k}}\right)-w_{\pi_{\ell_{k}+1}}\left(t_{e_{k}}\right) \geq \sum_{\pi_{\ell} \in F_{i j}(t)} w_{\pi_{\ell}}\left(t_{e_{k}}\right)-w_{\pi_{\ell+1}}\left(t_{e_{k}}\right) .
$$

The last line directly leads to

$$
\left(w_{\pi_{i}}(t)-w_{\pi_{j}}(t)\right)^{2} \geq \sum_{\pi_{\ell} \in F_{i j}(t)}\left(w_{\pi_{\ell}}\left(t_{e_{k}}\right)-w_{\pi_{\ell+1}}\left(t_{e_{k}}\right)\right)^{2}
$$

The second part of the proof now deals with finding a lower bound for $\sum_{i=2}^{n} d_{i}^{-}(t)+d_{i}(t)+d_{i}^{+}(t)$ in a fixed round $t$.

$$
\begin{aligned}
\sum_{i=2}^{n} d_{i}^{-}(t)+d_{i}(t)+d_{i}^{+}(t) & =\sum_{\left(\pi_{m}, \pi_{i}, \pi_{j}\right): \sigma_{m, i, j}(t)=1}\left(w_{\pi_{i}}(t)-w_{\pi_{j}}(t)\right)^{2} \\
& \geq \sum_{\left(\pi_{m}, \pi_{i}, \pi_{j}\right): \sigma_{m, i, j}(t)=1} \sum_{\pi_{\ell} \in F_{i j}(t)}\left(w_{\pi_{\ell}}\left(t_{e_{k}}\right)-w_{\pi_{\ell+1}}\left(t_{e_{k}}\right)\right)^{2} \\
& \geq \sum_{\pi_{\ell} \in L(t)}\left(w_{\pi_{\ell}}\left(t_{e_{k}}\right)-w_{\pi_{\ell+1}}\left(t_{e_{k}}\right)\right)^{2} .
\end{aligned}
$$

Lastly, we plug all insights together to conclude the proof.

$$
\begin{aligned}
\Phi\left(t_{e_{k}}\right)-\Phi\left(t_{e_{k+1}}\right) & \geq \frac{1}{4} \cdot \sum_{t=t_{e_{k}}}^{t_{e_{k+1}}} \sum_{i=2}^{n} d_{i}^{-}(t)+d_{i}(t)+d_{i}^{+}(t) \\
& \geq \frac{1}{4} \cdot \sum_{t=t_{e_{k}}}^{t_{e_{k+1}}} \sum_{\pi_{\ell} \in L(t)}\left(w_{\pi_{\ell}}\left(t_{e_{k}}\right)-w_{\pi_{\ell+1}}\left(t_{e_{k}}\right)\right)^{2} . \\
& =\frac{1}{4} \sum_{\pi_{\ell}=1}^{n-1}\left(w_{\pi_{\ell}}\left(t_{e_{k}}\right)-w_{\pi_{\ell+1}}\left(t_{e_{k}}\right)\right)^{2} .
\end{aligned}
$$

The last line follows since each robot moves at least once per epoch.

Lemma 5. Suppose that $\Phi\left(t_{e_{k}}\right)>0$. Then, $\frac{\Phi\left(t_{e_{k}}\right)-\Phi\left(t_{e_{k+1}}\right)}{\Phi\left(t_{e_{k}}\right)} \geq \frac{1}{8 n^{2}}$.

Proof. Lemma 4 leads to 


$$
\begin{aligned}
\frac{\Phi\left(t_{e_{k}}\right)-\Phi\left(t_{e_{k+1}}\right)}{\Phi\left(t_{e_{k}}\right)} & \geq \frac{1}{4} \frac{\sum_{\pi_{\ell}=1}^{n-1}\left(w_{\pi_{\ell}}\left(t_{e_{k}}\right)-w_{\pi_{\ell+1}}\left(t_{e_{k}}\right)\right)^{2}}{\sum_{\pi_{\ell}=1}^{n}\left(w_{\pi_{\ell}}\left(t_{e_{k}}\right)-1\right)^{2}} \\
& =\frac{1}{4} \frac{\sum_{\pi_{\ell}=1}^{n-1}\left(w_{\pi_{\ell}}\left(t_{e_{k}}\right)-w_{\pi_{\ell+1}}\left(t_{e_{k}}\right)\right)^{2}}{\sum_{\pi_{\ell}=1}^{n}\left(w_{\pi_{\ell}}\left(t_{e_{k}}\right)-w_{\pi_{1}}\left(t_{e_{k}}\right)\right)^{2}} .
\end{aligned}
$$

The second line follows since $w_{1}(t)=1$ for all $t$ and thus $w_{\pi_{1}}\left(t_{e_{k}}\right)=1$. Observe that the righthand side does not change if we multiply each $w_{\pi_{i}}\left(t_{e_{k}}\right)$ with the same constant. Additionally, it also does not change if we add the same constant to each $w_{\pi_{i}}\left(t_{e_{k}}\right)$. Hence, we can assume w.l.o.g. $\sum_{\pi_{\ell}=1}^{n} w_{\pi_{\ell}}\left(t_{e_{k}}\right)=0$ and $\sum_{\pi_{\ell}=1}^{n}\left(w_{\pi_{\ell}}\left(t_{e_{k}}\right)-w_{\pi_{1}}\left(t_{e_{k}}\right)\right)^{2}=1$ and obtain

$$
\frac{\Phi\left(t_{e_{k}}\right)-\Phi\left(t_{e_{k+1}}\right)}{\Phi\left(t_{e_{k}}\right)} \geq \frac{1}{4} \min _{\substack{w_{1} \geq w_{2}, \ldots, \geq w_{n} \\ \sum_{i} w_{i}=0 \\ \sum_{i}\left(w_{i}-w_{1}\right)^{2}=1}} \sum_{i=1}^{n-1}\left(w_{i}-w_{i+1}\right)^{2}
$$

The assumption $\sum_{i}\left(w_{i}-w_{1}\right)^{2}=1$ implies that the average value of all $\left(w_{i}-w_{1}\right)^{2}$ is $\frac{1}{n}$ and hence there is at least some $j$ with $\left|w_{j}-w_{1}\right| \geq \frac{1}{\sqrt{n}}$. As a consequence, either $\left|w_{1}\right| \geq \frac{1}{2 \cdot \sqrt{n}}$ or $\left|w_{j}\right| \geq \frac{1}{2 \cdot \sqrt{n}}$. W.l.o.g. assume $\left|w_{1}\right| \geq \frac{1}{2 \sqrt{n}}$ and moreover assume $w_{1}>0$. The case $w_{1}<0$ can be handled by multiplying each $w_{i}$ with -1 and sort the elements in descending order.

Now define $u_{i}=w_{i}-w_{i+1}$ for $i<n$ and $u_{n}=0$. It holds $u_{i} \geq 0$ for all $i$ and $\sum_{i=1}^{n} u_{i}=w_{1}-w_{n}$. Since at least one $w_{1} \geq \frac{1}{2 \sqrt{n}}$ and the $\sum_{i} w_{i}=0$, we can conclude $u_{n}<0$ and thus $\sum_{i} u_{i} \geq \frac{1}{2 \sqrt{n}}$.

As a final step, we obtain

$$
\frac{\Phi\left(t_{e_{k}}\right)-\Phi\left(t_{e_{k+1}}\right)}{\Phi\left(t_{e_{k}}\right)} \geq \frac{1}{4} \min _{u_{i} \geq 0, \sum_{i} u_{i} \geq 1 /(2 \sqrt{n})} \sum_{i=1}^{n-1} u_{i}^{2} .
$$

The solution of the minimization problem is $u_{i}=\frac{1}{2 \cdot n^{3 / 2}}$ for each $i$.

Hence,

$$
\frac{\Phi\left(t_{e_{k}}\right)-\Phi\left(t_{e_{k+1}}\right)}{\Phi\left(t_{e_{k}}\right)} \geq \frac{1}{4} \cdot \frac{1}{2 \cdot n^{2}}=\frac{1}{8 n^{2}}
$$

Lemma 11. After $\mathcal{O}\left(n^{2} \cdot \log (n / \varepsilon)\right)$ rounds, it holds $\sum_{i=2}^{n} w_{i}(t) \geq(1-\varepsilon) \cdot(n-1)$.

Proof. Fix any epoch $e_{k}$. By Lemma 5, we obtain $\Phi\left(t_{e_{k+1}}\right) \leq\left(1-\frac{1}{8 n^{2}}\right) \cdot \Phi\left(t_{e_{k}}\right)$ and thus $\Phi\left(t_{e_{k+x}}\right) \leq$ $\left(1-\frac{1}{8 n^{2}}\right)^{x} \cdot \Phi\left(t_{e_{k}}\right)$. Observe that $(1-y)^{x} \leq e^{-y \cdot x}$ where $e$ denotes Euler's number. Thus, choosing $x \geq 8 n^{2} \cdot \ln \left(\frac{1}{r}\right)$ yields $\Phi\left(t_{e_{k+x}}\right) \leq r \cdot \Phi\left(t_{e_{k}}\right)$. Since $\Phi\left(t_{e_{k}}\right)<n-1, r \leq \frac{\varepsilon}{n-1}$ leads to $\Phi\left(t_{e_{k+x}}\right) \leq \varepsilon$ and thus $\sum_{i=2}^{n} w_{i}(t) \geq(1-\varepsilon) \cdot(n-1)$. 


\section{Adjusted $\mathcal{F}$ sync Algorithm}

The $\mathcal{F}_{\text {SYNC algorithm presented in Section 5.1 }}$ is - to keep the pseudocode comprehensible - designed such that the robots still move to the left after MAX-LinE-Formation is already solved. In this section, we explain how 3 additional lights help to remove this behavior to design an algorithm that forms a stationary line.

Observe first that in Algorithm 2, two runs can only be located at two neighboring robots in case the algorithm is already in phase 2. Otherwise, at least one robot observes that its neighborhood is not yet aligned parallel to the $y$-axis, and the corresponding run is stopped (line 31 in Algorithm 2. We use this observation as follows: As soon as two runs meet at neighboring robots, the two robots activate a light $\ell_{\text {final }}$ to store this information. Robots with an active light $\ell_{\text {final }}$ do not move to the left anymore. Additionally, robots that observe a robot in their neighborhood that has activated $\ell_{\text {final }}$, activate their own light $\ell_{\text {final }}$. Hence, after $\mathcal{O}(n)$ rounds, all robots have activated $\ell_{\text {final }}$. While propagating $\ell_{\text {final }}$, it might, however, happen that some robots move to the left while other robots remain stationary (due to the limited visibility). See Figure 6 for a depiction of such a case. To rebuild the line-shape again runs at robots with active light $\ell_{\text {final }}$ behave slightly different: the vertical movement is identical to before. The horizontal movement changes: instead of moving to the left, a robot moves a distance of 1 to the right if it is leftmost in its neighborhood, and there is

at least one robot in a horizontal distance of 1 to the right. Finally, the robots align again on the initial line (before activating $\ell_{\text {final }}$ ) and MAX-Line-Formation gets solved after $\mathcal{O}(n)$ rounds. 


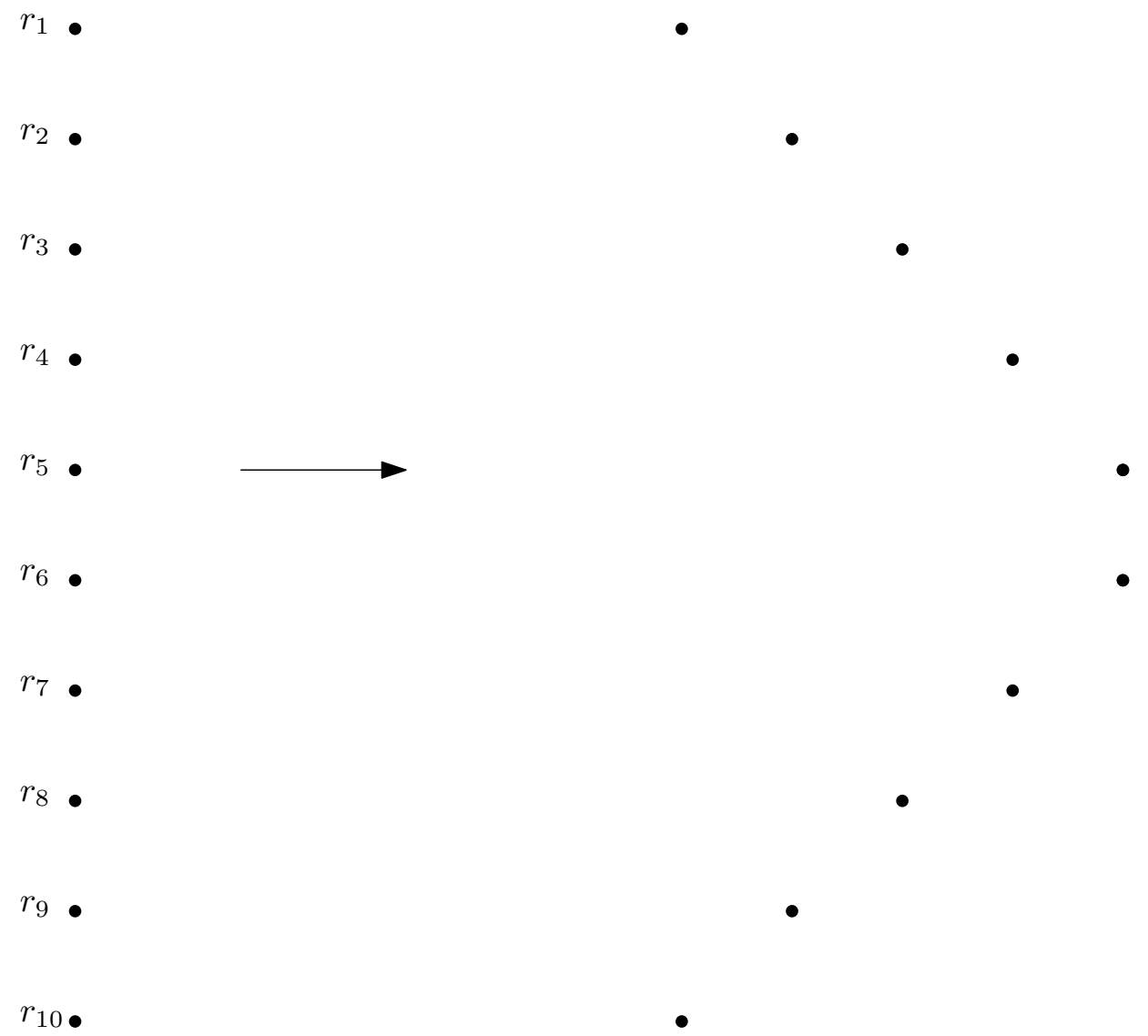

Figure 6: To the left, the robots are arranged on a straight line parallel to the $y$-axis. After some time, two runs meet in the middle at $r_{5}$ and $r_{6}$ that activate their light $\ell_{\text {final }}$. In the next round, $r_{4}$ and $r_{7}$ activate their light $\ell_{\text {final }}$ and so on. However, $r_{1}, r_{2}, r_{3}, r_{4}, r_{7}, r_{8}, r_{9}$ and $r_{10}$ move a distance of 1 to the left before $r_{4}$ and $r_{7}$ become stationary. Similarly, $r_{1}, \ldots, r_{3}$ and $r_{8}, \ldots, r_{10}$ move a distance of 1 further to the left than $r_{3}$ and $r_{6}$ and so on. The final configuration might look like it is depicted to the right. 


\section{Omitted Proofs of Section 5}

Lemma 6. After $\mathcal{O}(n)$ epochs, all robots are located on distinct positions on the same vertical line parallel to the $y$-axis. Moreover, the configuration is connected.

Proof. The connectivity and collision avoidance follow directly from the algorithm description. To prove the linear runtime, define $x_{\max }(t)$ to be the maximal $x$-coordinate in the global coordinate system. Furthermore, define $k_{\max }(t)$ to be the number of robots with $x_{i}(t) \in\left(x_{\max }(t)-1, x_{\max }(t)\right]$. Observe that every robot $r_{i}$ with $x_{i}(t) \in\left(x_{\max }(t)-1, x_{\max }(t)\right]$ moves such that $x_{i}(t+1) \in\left(x_{\max }(t)-\right.$ $\left.2, x_{\max }(t)-1\right]$. Since the configuration is always connected, there must have been a robot $r_{j}$ with $x_{j}(t) \in\left(x_{\max }(t)-2, x_{\max }(t)-1\right]$ that cannot leave the interval. It follows $k_{\max }(t+1) \geq k_{\max }(t)+1$ Thus, after $\mathcal{O}(n)$ rounds, all robots have $x$-coordinates in an interval of size at most 1 . Fix one round $t$ and assume that all robots have $x$-coordinates in an interval of size at most 1 . Consider the robot $r_{\max }$ with globally maximal $x$-coordinate. None of its neighbors can see a robot that has a larger $x$-coordinate, and thus, all robots of $N_{\max }(t)$ are collinear in round $t+1$. Furthermore, all of these robots still have the globally largest $x$-coordinate in round $t+1$. Now, consider the topmost robot $r_{j} \in N_{\max }(t)$. It follows that all robots of $N_{j}(t+1)$ have a smaller or equal $x$-coordinate and cannot see any other robot with a larger $x$-coordinate. Thus, in round $t+2$ all robots in $N_{\max }(t)$ and $N_{j}(t+1)$ are collinear. The same argument holds for the bottom-most robot. Applying the same argument inductively yields that all robots are collinear after $\mathcal{O}(n)$ rounds.

Theorem 7. After $\mathcal{O}(n)$ epochs, the robots have solved MaX-Line-Formation.

Proof. By Lemma 6 all robots are collinear on a line parallel to the $y$-axis after $\mathcal{O}(n)$ epochs. It remains to prove the linear runtime until the optimal configuration is formed. Rename the robots such that $r_{1}$ is the topmost robot and $r_{n}$ is the bottom-most robot. Define $u_{i}(t)=y_{i}(t)-y_{i-1}(t)$. Both $r_{1}$ and $r_{n}$ activate their light $\ell_{\text {mov }}$ every 3 rounds and ensure $u_{2}(t)=u_{n}(t)=1$. In round $t+1$ it holds $u_{3}(t+1)=u_{n-1}(t+1)=1$ and so on. Assume $n$ to be even (the arguments for odd $n$ are analogous). After $\frac{n}{2}-1$ rounds, the movement meets at the two robots $r_{n / 2}$ and $r_{n / 2+1}$ and they move such that $y_{n / 2+1}\left(t+\frac{n}{2}-1\right)=1$ holds. The next two movements ensure $y_{n / 2}\left(t+\frac{n}{2}+2\right)=y_{n / 2+2}\left(t+\frac{n}{2}+2\right)=1$ and so on. Since each 3 rounds a new movement is started, the optimal configuration is reached in $\mathcal{O}(n)$ rounds. 


\section{E Omitted Proofs of Section 6}

Lemma 12. After $\mathcal{O}(\Delta)$ epochs, all robots are located on the same vertical line parallel to the $y$-axis. Moreover, the configuration is connected.

Proof. Define by $x_{\min }(t)$ the minimal $x$-coordinate of all robots. Next, define intervals $i_{1}(t)=$ $\left[x_{\min }(t)+1\right], i_{2}(t)=\left[x_{\min }(t)+1, x_{\min }(t)+2\right]$ and so on. Additionally, $R_{j}(t):=\left\{r_{k} \mid x_{k}(t) \in i_{j}(t)\right\}$ and $k(t)$ is the largest index $j$ of an interval such that $R_{j}(t) \neq \emptyset$. Observe that $k(t) \leq \Delta_{x}$. Fix a round $t_{0}$. By the same arguments as in the proof of Lemma 6 , it holds $R_{k\left(t_{0}\right)}\left(t_{0}+1\right)=\emptyset$ (since all robots leave the rightmost interval). At the same time, it can happen that some of the robots in the leftmost interval $i_{1}\left(t_{0}\right)$ create a new interval to the left such that $i_{1}\left(t_{0}+1\right) \neq i_{1}\left(t_{0}\right)$. In total, however, at most $\Delta_{y}$ new intervals can be created since for every new interval, it must hold that the robots cannot observe the position of any of the previous new generated intervals. Since initially at most $\Delta_{x}$ intervals exist, we obtain a runtime of $\Delta_{x}+\Delta_{y} \in \mathcal{O}(\Delta)$ until all robots have an $x$-coordinate in the same interval. Now, consider the robot $r_{\max }$ with globally maximal $x$-coordinate. None of its neighbors can see a robot that has a larger $x$-coordinate, and thus, all robots of $N_{\max }(t)$ are collinear in round $t+1$. Furthermore, all of these robots still have the globally largest $x$-coordinate in round $t+1$. Next, consider the topmost robot $r_{j} \in N_{\max }(t)$. It follows that all robots of $N_{j}(t+1)$ have a smaller or equal $x$-coordinate and cannot see any other robot with a larger $x$-coordinate. Thus, in round $t+2$ all robots in $N_{\max }(t)$ and $N_{j}(t+1)$ are collinear. The same argument holds for the bottom-most robot. This case can occur at most $\Delta_{y}$ times. Afterward, all robots are collinear. The runtime of $\mathcal{O}(\Delta)$ follows.

Theorem 8. GATHERING of $n$ robots agreeing on one axis of their local coordinate systems in the $\mathcal{O B L O T}$ model can be solved in $\mathcal{O}(\Delta)$ epochs under the $\mathcal{F}_{\mathrm{SYNC}}$ scheduler.

Proof. According to Lemma 12, all robots are collinear after $\mathcal{O}(\Delta)$ rounds. Rename the robots such that $r_{1}$ is the topmost robot and $r_{n}$ the bottom-most robot. We prove exemplary for $r_{1}$ that it moves a constant distance toward $r_{n}$ every two rounds. The arguments for $r_{n}$ are analogous. Observe first that $r_{1}$ remains topmost because $r_{1}$ moves to the midpoint between its position and its farthest neighbor $r_{f}$. In case there is any robot between $r_{1}$ and $r_{f}$, this robot can also see both $r_{1}$ and $r_{f}$ and either moves to the same position as $r_{1}$ or can see a robot $r_{f^{\prime}}$ that lies below of $r_{f}$. Similarly, $r_{f}$ and robots below of $r_{f}$ can only compute target points below the target point of $r_{1}$. Hence, $r_{1}$ remains the topmost robot. Now consider a round in which $r_{1}$ moves a distance of less than $\frac{1}{10}$ downwards. This implies that its farthest neighbor $r_{f}$ is in distance at most $\frac{1}{5}$. Since the configuration is connected, $r_{f}$ can see a robot $r_{f^{\prime}}$ in distance at least 1 of $r_{1}$. Hence, $r_{1}$ moves a distance of at least $\frac{1}{2}$ downwards. Thus, the distance between $r_{1}$ and $r_{f}$ in round $t+1$ is at least $\frac{1}{2}$. Hence, $r_{1}$ moves a constant distance (at least $\frac{1}{4}$ ) in round $t+2$. Thus, every two rounds, $r_{1}$ and $r_{n}$ move at least a constant distance. Finally, they can see each other, and all robots gather in the next round.

\section{E.1 Proof of Theorem 9}

Lemma 13. For any round $t$, it holds

$$
\Phi(t+1)=\Phi(t)-\frac{1}{4} \sum_{i=2}^{n} d_{i}^{-}(t)+d_{i}(t)+d_{i}^{+}(t) .
$$

Proof. Analogous to the proof of Lemma 10. 
Next, define $w_{\pi_{1}}\left(t_{e_{k}}\right), w_{\pi_{2}}\left(t_{e_{k}}\right), \ldots, w_{\pi_{n}}\left(t_{e_{k}}\right)$ to the values $w_{1}^{x}(t), \ldots, w_{n+1}^{x}(t)$ sorted from largest to smallest with ties broken arbitrarily.

Lemma 14. For any epoch $k$, it holds

$$
\Phi\left(t_{e_{k}}\right)-\Phi\left(t_{e_{k+1}}\right) \geq \frac{1}{4} \sum_{i=1}^{n-1}\left(w_{\pi_{i}}(t)-w_{\pi_{i+1}}(t)\right)^{2} .
$$

Proof. Analogous to the proof of Lemma 4.

Lemma 15. Suppose that $\Phi\left(t_{e_{k}}\right)>0$. Then,

$$
\frac{\Phi\left(t_{e_{k}}\right)-\Phi\left(t_{e_{k+1}}\right)}{\Phi\left(t_{e_{k}}\right)} \geq \frac{1}{4(n+1)^{2}}
$$

Proof. Lemma 4 leads to

$$
\frac{\Phi\left(t_{e_{k}}\right)-\Phi\left(t_{e_{k+1}}\right)}{\Phi\left(t_{e_{k}}\right)} \geq \frac{1}{4} \frac{\sum_{\pi_{\ell}=1}^{n}\left(w_{\pi_{\ell}}\left(t_{e_{k}}\right)-w_{\pi_{\ell+1}}\left(t_{e_{k}}\right)\right)^{2}}{\sum_{\pi_{\ell}=1}^{n+1}\left(w_{\pi_{\ell}}\left(t_{e_{k}}\right)-\bar{x}\right)^{2}}
$$

Observe that the right-hand side does not change if we multiply each $w_{\pi_{i}}\left(t_{e_{k}}\right)$ with the same constant. Additionally, it also does not change if we add the same constant to each $w_{\pi_{i}}\left(t_{e_{k}}\right)$. Hence, we can assume w.l.o.g. $\sum_{\pi_{\ell}=1}^{n+1} w_{\pi_{\ell}}\left(t_{e_{k}}\right)=0$ and $\sum_{\pi_{\ell}=1}^{n+1}\left(w_{\pi_{\ell}}\left(t_{e_{k}}\right)\right)^{2}=1$ and obtain

$$
\frac{\Phi\left(t_{e_{k}}\right)-\Phi\left(t_{e_{k+1}}\right)}{\Phi\left(t_{e_{k}}\right)} \geq \frac{1}{4} \min _{\substack{w_{1} \geq w_{2}, \ldots, \geq w_{n+1} \\ \sum_{i} w_{i}=0 \\ \sum_{i}\left(w_{i}-w_{1}\right)^{2}=1}} \sum_{i=1}^{n}\left(w_{i}-w_{i+1}\right)^{2}
$$

The assumption $\sum_{i} w_{i}^{2}=1$ implies that the average value of all $w_{i}^{2}$ is $\frac{1}{n}$ and hence there is at least some $j$ with $\left|w_{j}\right| \geq \frac{1}{\sqrt{n}}$. W.l.o.g. assume this $w_{j}$ is positive. The case $w_{J}<0$ can be handled by multiplying each $w_{i}$ with -1 and sorting the elements in descending order.

Now define $u_{i}=w_{i}-w_{i+1}$ for $i<n+1$ and $u_{n+1}=0$. It holds $u_{i} \geq 0$ for all $i$ and $\sum_{i=1}^{n} u_{i}=w_{1}-w_{n+1}$. Since $w_{j} \geq \frac{1}{\sqrt{n}}$ and $\sum_{i} w_{i}=0$, we can conclude $u_{n+1}<0$ and thus $\sum_{i} u_{i} \geq \frac{1}{\sqrt{n}}$.

As a final step, we obtain

$$
\frac{\Phi\left(t_{e_{k}}\right)-\Phi\left(t_{e_{k+1}}\right)}{\Phi\left(t_{e_{k}}\right)} \geq \frac{1}{4} \min _{u_{i} \geq 0, \sum_{i} u_{i} \geq 1 /(2 \sqrt{n})} \sum_{i=1}^{n+1} u_{i}^{2} .
$$

The solution of the minimization problem is $u_{i}=\frac{1}{(n+1)^{3 / 2}}$ for each $i$.

Hence,

$$
\frac{\Phi\left(t_{e_{k}}\right)-\Phi\left(t_{e_{k+1}}\right)}{\Phi\left(t_{e_{k}}\right)} \geq \frac{1}{4} \cdot \frac{1}{(n+1)^{2}}
$$


Proof of Theorem 9. Fix any epoch $e_{k}$. By Lemma 15, we obtain $\Phi\left(t_{e_{k+1}}\right) \leq\left(1-\frac{1}{4 n^{2}}\right) \cdot \Phi\left(t_{e_{k}}\right)$ and thus $\Phi\left(t_{e_{k+x}}\right) \leq\left(1-\frac{1}{4 n^{2}}\right)^{x} \cdot \Phi\left(t_{e_{k}}\right)$. Observe that $(1-y)^{x} \leq e^{-y \cdot x}$ where $e$ denotes Euler's number. Thus, choosing $x \geq 4(n+1)^{2} \cdot \ln \left(\frac{1}{r}\right)$ yields $\Phi\left(t_{e_{k+x}}\right) \leq r \cdot \Phi\left(t_{e_{k}}\right)$. Since $\Phi\left(t_{e_{k}}\right)<n+1, r \leq \frac{\varepsilon}{n+1}$ leads to $\Phi\left(t_{e_{k+x}}\right) \leq \varepsilon$ and the theorem follows. 